\title{
A review of the studies on nonvisual lighting effects in the field of physiological anthropology
}

\author{
Tetsuo Katsuura ${ }^{1 *}$ (D) and Soomin Lee ${ }^{2}$
}

\begin{abstract}
Here, we review the history and the trends in the research on the nonvisual effect of light in the field of physiological anthropology. Research on the nonvisual effect of light in the field of physiological anthropology was pioneered by Sato and colleagues in the early 1990s. These authors found that the color temperature of light affected physiological functions in humans. The groundbreaking event with regard to the study of nonvisual effects of light was the discovery of the intrinsically photosensitive retinal ganglion cells in the mammalian retina in the early 2000s. The interest of the physiological anthropology scientific community in the nonvisual effects of light has been increasing since then. A total of 61 papers on nonvisual effects of light were published in the Journal of Physiological Anthropology (including its predecessor journals) until October 2018, 14 papers (1.4/year) in the decade from 1992 to 2001, 45 papers (2.8/year) in the 16 years between 2002 and 2017, and two papers in 2018 (January-October). The number of papers on this topic has been increasing in recent years. We categorized all papers according to light conditions, such as color temperature of light, light intensity, and monochromatic light. Among the 61 papers, 11 papers were related to color temperature, 20 papers were related to light intensity, 18 papers were related to monochromatic light, and 12 papers were classified as others. We provide an overview of these papers and mention future research prospects.
\end{abstract}

Keywords: Light, Nonvisual effect, Physiological anthropology, Color temperature, Light intensity, Monochromatic light, Intrinsically photosensitive retinal ganglion cell, Pupillary light, Melatonin suppression

\section{Background}

Light has a great influence on various organisms, including humans. Photoreceptors or photopigments, which accept light from the surrounding environment, are present not only in animals and plants, but also in bacteria and fungi, and are involved in several life-related processes. For example, phytochrome is a chromoprotein contained in plants, cyanobacteria, bacteria, and even in fungi, which accepts red light and far-red light [1]. In higher plants, phytochrome is involved in the regulation of flowering, germination, and shade avoidance reaction [2].

Rhodopsin is a vertebrate photopigment and is expressed in the rods. Opsins are photopigments that respond to red, green, blue, and violet and are present in the cones. Phylogenetically, red opsin (LWS) is the oldest photopigment

\footnotetext{
* Correspondence: katsu@faculty.chiba-u.jp

${ }^{1}$ Graduate School of Engineering, Chiba University, 1-33 Yayoi-cho, Inage-ku,

Chiba 263-8522, Japan

Full list of author information is available at the end of the article
}

followed by violet opsin (SWS1), blue opsin (SWS2), and green opsin (RH2). Finally, rhodopsin (RH1) was evolved from the green opsin [3]. During evolution, early mammals lost the green and blue opsins, which resulted in a loss of color vision in their nocturnal lifestyle. This is because, in nocturnal environments, color vision is not essential to survival, and it is more important to maximize the amount of light one can capture, rather than distinguish among spectral wavelengths [4]. However, in the diurnal primates, a mutation in the red opsin led to an alternate form of the green opsin [5]. Humans have violet (customarily called "blue"), green, and red opsins and rhodopsin. The human vision can perceive three colors (trichromacy) and is scotopic [6]. In addition to these visually related photopigments, an opsin (melanopsin, OPN4) is expressed in the mammalian intrinsically photosensitive retinal ganglion cells (ipRGCs) and is involved in various nonvisual or nonimage-forming (NIF) responses, such as adjustment of circadian rhythm and pupillary constriction [7-9]. Melanopsin

(c) The Author(s). 2019 Open Access This article is distributed under the terms of the Creative Commons Attribution 4.0 International License (http://creativecommons.org/licenses/by/4.0/), which permits unrestricted use, distribution, and 
received its name because it was firstly identified in frog's melanocyte [10]. Nevertheless, we know it now be closer to invertebrate's rather than vertebrate's photopigments $[9,11]$.

Physiological anthropology is one branch of anthropology and an academic discipline devoted to study the human biological nature from a physiological point of view. Ultimately, it aims to use this knowledge to improve people's quality of life [12]. Research on physiological anthropology in Japan began with the study on the functions of the skeletal muscles and of the central nervous system in the 1950s. Since then, the research topics addressed have been expanding to several fields including human adaptability to the environment and human variability [12]. A large number of studies on environmental adaptability have been conducted in regard to the thermal environmenthow has the human organism adapted to hot and cold environments? In the last $10-20$ years, taking into consideration the developments on photopigments, there has been increasing interest around the light environment as well, especially in regard to nonvisual responses to light.

Herein, we outline the history and discuss the trends in the research on the nonvisual effect of light in the field of physiological anthropology. We mainly searched and reviewed the papers published in the Journal of Physiological Anthropology (JPA) and its predecessor journals (The Annals of Physiological Anthropology, Applied Human Science, Journal of Physiological Anthropology and Applied Human Science). We focused on JPA since it is the only journal on physiological anthropology in the world. We believe it may then constitute the most suitable source allowing to gather information about the current stateof-art information on this topic. However, it should be noted that the JPA does not include all studies from the perspective of the physiological anthropology field.

\section{The primordia of the research on nonvisual effects of light in the field of physiological anthropology}

Research on the nonvisual effect of light in the field of physiological anthropology was begun by Sato et al. in the early 1990s; they mainly studied the influence of color temperature of light on human physiology, as measured by parameters such as the contingent negative variation (CNV) [13], blood pressure and critical flicker frequency [14], and heart rate variability (HRV) [15]. In 1941, Kruithof [16], a Dutch physicist, examined the influence of illuminance and color temperature on human pleasantness. Although he did not describe his methodological approach in detail, he might have not included any sort of physiological measurements. When Sato and colleagues began their research, the literature of architecture, illumination engineering, and ergonomics in Japan considered that the color temperature of the light should be no less than $4000 \mathrm{~K}$, with desirable targets of more than $4500 \mathrm{~K}$. Thus, a high color temperature of light was recommended. Sato wondered about this situation; at the same time, he had been asked to conduct research on this topic by a fluorescent light manufacturer. Therefore, he started conducting these studies to determine an optimal color temperature of light for humans. In these studies, Sato and colleagues found that CNV [13], diastolic blood pressure [14], and both Mayer-wave related sinus arrhythmia and respiratory sinus arrhythmia increased [15] in response to a high color temperature environment, when compared with a low color temperature environment. Bearing in mind that the work performance did not seem to improve under high color temperature, they clarified that the high color temperature lighting was not always desirable [13]. Supporting this notion, they found the level of activity of the sympathetic nervous system to be lower in a low color temperature environment [14], which made them to develop the idea that light with these characteristics may in fact be considered comforting light. These studies by Sato and colleagues pioneered the first observations in the laboratory experiments that differences in spectroscopic spectrum of light, which can be expressed by the color temperature, affect the physiological functions in humans. They greatly contributed to the subsequent development of the research on the nonvisual effects of light in the field of physiological anthropology.

Particularly groundbreaking in the field was the discovery of ipRGC in the mammalian retina in the early 2000s. The hypothesis of a third class of photoreceptor was raised in the 1990s and derived from the observation that retinal degenerate mice, which lost their cones and rods, still showed normal circadian responses to light [17]. The novel opsin, melanopsin, was identified in the retinal cells of Xenopus laevis (African clawed frog) [11] in 1998. The third class, non-cone, and non-rod photoreceptor in the retina of mammals was discovered by two research groups, Berson et al. [7] and Hatter et al. [18] in 2002. They found a small subset of retinal ganglion cells that were intrinsically photosensitive $[7,18]$. Therefore, these cells were called intrinsically photosensitive retinal ganglion cells or ipRGCs $[19,20]$, which project to brain nuclei involved in nonvisual responses to light, such as the pupillary light reflex/ response (PLR) and the circadian photoentrainment [21]. The photosensitivity of these cells depends on the presence of melanopsin [7, 18-22]. Hence, these cells are also called melanopsin-expressing retinal ganglion cells (mRGCs) [23].

The discovery of ipRGC in the mammalian retina by Berson et al. [7] and Hatter et al. [18] had a strong impact on the study on nonvisual effects of light and was one of the main driving forces to increase research interested on the topic within the physiological anthropology community. Remarkably, a total of 61 papers on 
the nonvisual effects of light were published in the JPA (including its predecessor journals) by October 2018, 14 papers (1.4/year) in the decade from 1992 to 2001, 45 papers (2.8/year) in the 16 years from 2002 to 2017, and two papers in 2018 (January-October) (Fig. 1). This number has been increasing in recent years, which tacitly shows the increasing interest in this area of research. The proportion of papers on the nonvisual effects of light to the total number of papers published in JPA in this period are shown in Fig. 2. The percentages were varying between 0 to $6.9 \%$ of the total papers in each year and $3.6 \%$ on average in the first decade. Since 2002, the proportion has increased, with percentages varying between 0 to $20.0 \%$ yearly and an average of $6.7 \%$.

Herein, we classified each paper according to the light parameters explored in each manuscript, namely the color temperature of light, the light intensity, and the monochromatic light. We discuss in separate sections the evidence for each of these parameters. Those papers on both color temperature and light intensity were classified as color temperature, and those on both monochromatic light and light intensity, as monochromatic light. Field studies, laboratory experiments that did not use specified experimental light settings, and review articles widely discussing the nonvisual effects of light were classified as others. Figure 3 shows the distribution of the number of papers published in the JPA, from 1992 to 2018, according to the parameters of light inspected. Among all 61 papers, 11 papers were related to color temperature, 20 papers were related to light intensity, 18 papers were related to monochromatic light, and 12 papers were classified as others. As shown in Fig. 3, most papers on color temperature were published in the first half and in the middle of this period, with a more recent decline. Conversely, the number of papers examining the monochromatic light has been gradually increasing over time. After ipRGC discovery, monochromatic light has been considered an important topic of research. One to three papers on light intensity have been published yearly over a 15-year period.

We discuss below, in separate sections, the papers on color temperature, light intensity, monochromatic light, and others, as per the structure outlined above.

Nonvisual effects of the color temperature of light The influence of the color temperature of light on human physiology was the first aspect researched in the field of physiological anthropology and began, as previously mentioned, in the early 1990s [13-15]. Since then, this topic has been actively studied in the field of physiological anthropology (Table 1). Effects of color temperature of light exposed before sleeping on body temperature and melatonin secretion during sleep were examined. It was reported that exposure to high color temperature light before sleep tended to suppress the decrease in body temperature and the melatonin secretion levels during sleep [24]. Another study has focused on the effects of color temperature on sleep stage, as measured by polysomnographic recordings. This study found the amount

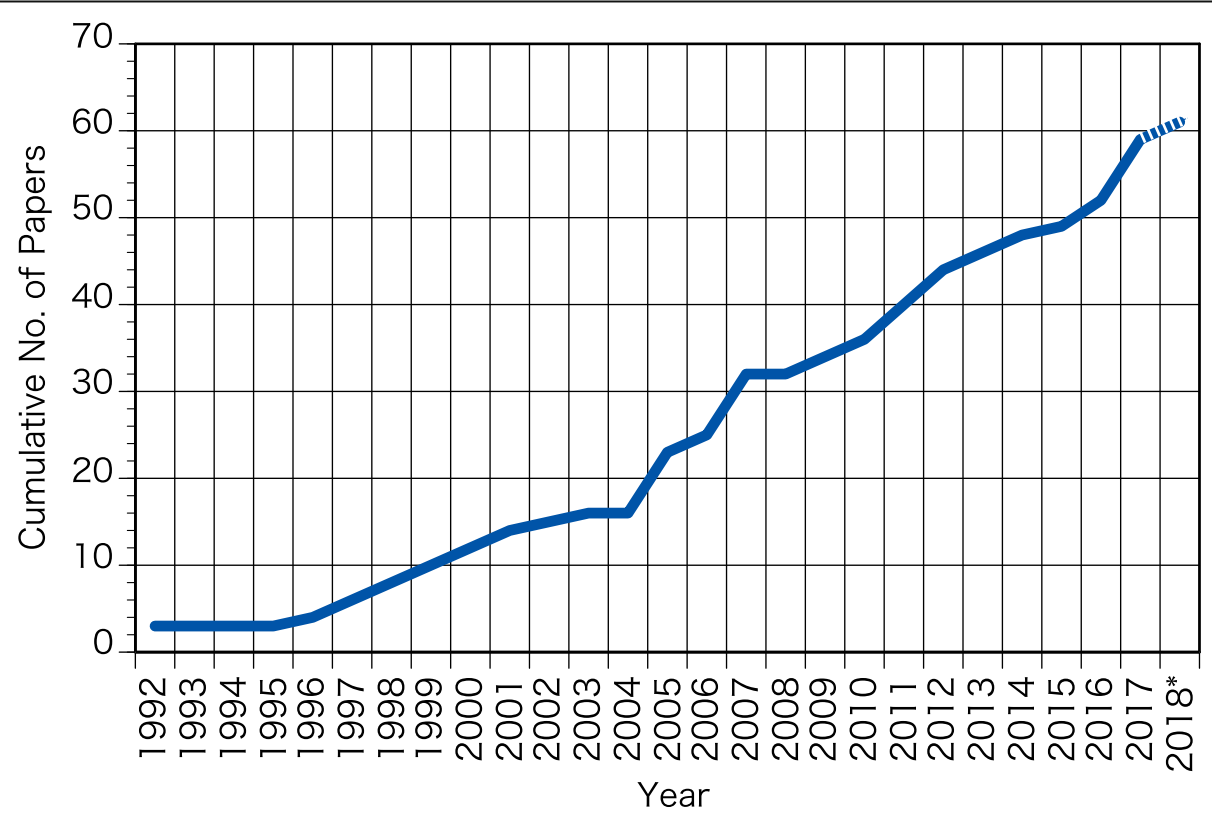

Fig. 1 Cumulative number of papers on the nonvisual effects of light published in the JPA from 1992 to $2018^{*}$ (asterisk denotes data from January to October were obtained in 2018) 


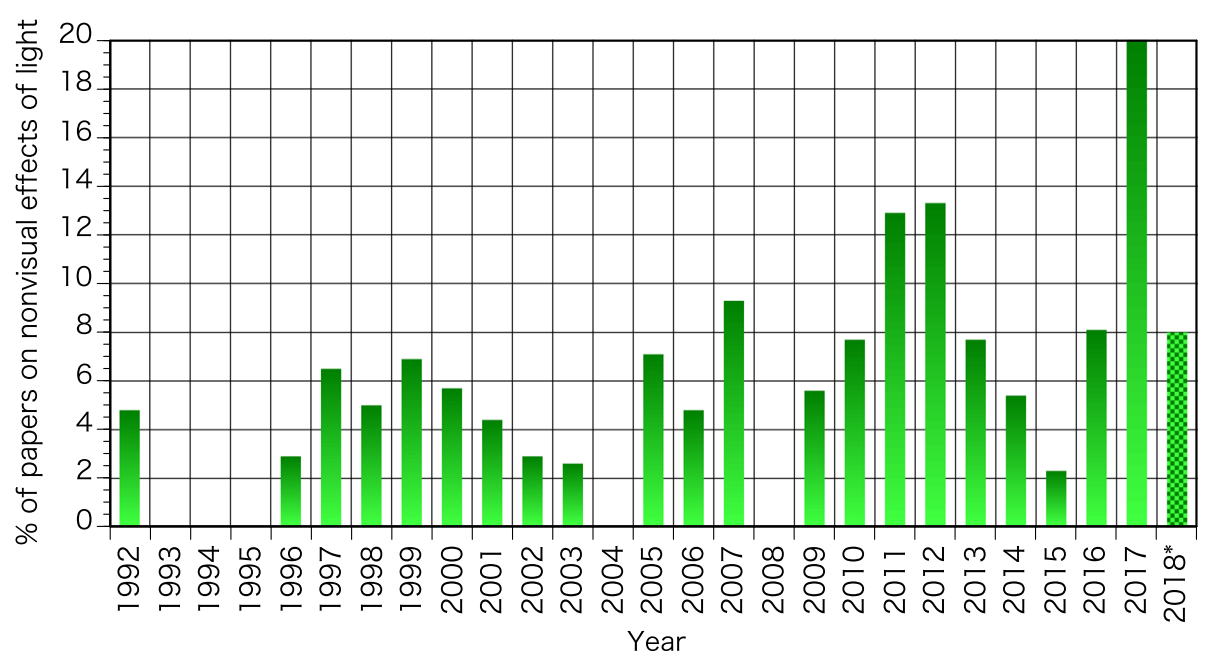

Fig. 2 Proportion of papers on the nonvisual effects of light to the total number of papers published in the JPA from 1992 to $2018^{*}$ (asterisk denotes data from January to October were obtained in 2018)

of stage 4 sleep to be attenuated in the early phase of sleep [25]. High color temperature light was also found to attenuate the suppressions of heart rate observed during sleep and to increase the high-frequency (HF) components of HRV during sleep [26]. Altogether, these studies suggest that exposure to high color temperature light before sleep may be detrimental.

One study has specifically evaluated physiological functions during short-term (22 $\mathrm{min})$ exposure to light of different color temperatures and subsequently short-term (20 min) sleep after exposure [27]. It was found that the alpha attenuation coefficient (AAC) and mean power frequency of electroencephalogram (EEG) were lower during low color temperature light exposure, when compared with high color temperature. These findings suggest that low color temperature light is able to create a smooth lowering of central nervous system activity [27].

In respect to the influence of color temperature on body temperature regulation, it was found that lower color temperature environment enhanced the decrease in mean skin temperature when the air temperature was decreased from 28 to $18{ }^{\circ} \mathrm{C}$ and in a cold environment $\left(15^{\circ} \mathrm{C}\right)$. Evaluations of the rectal temperature confirmed this trend [28]. We have examined the influences of

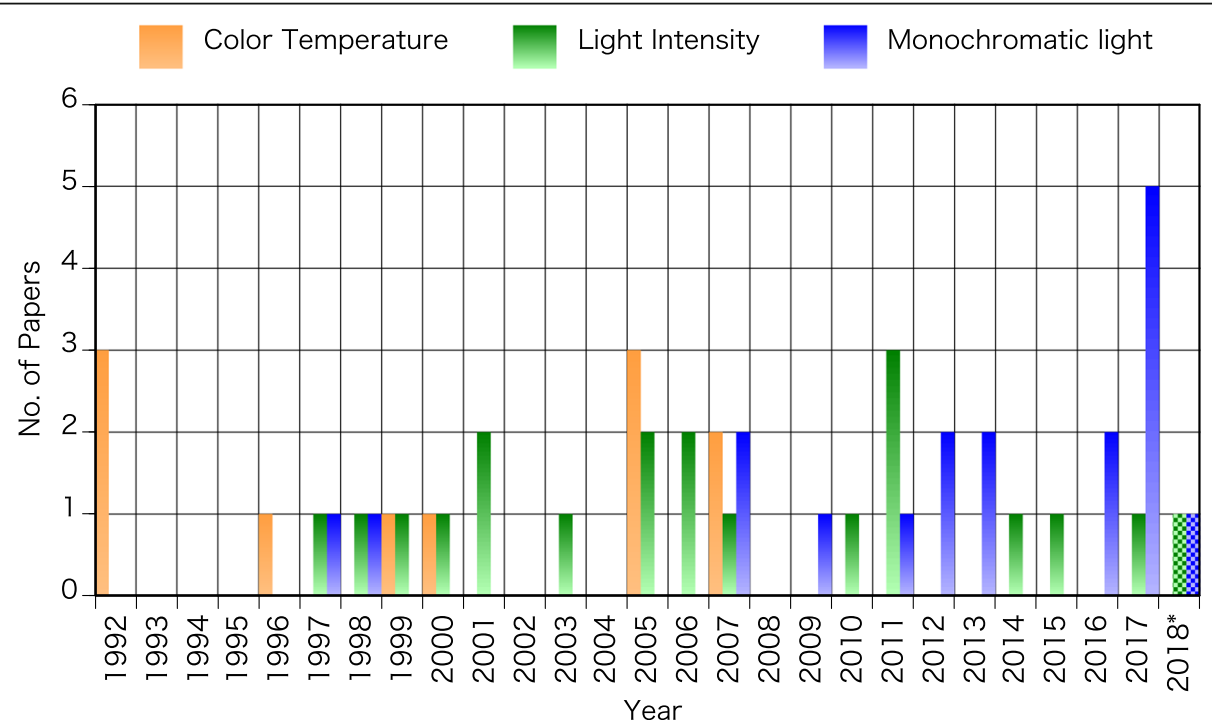

Fig. 3 Number of papers for each lighting conditions published in the JPA from 1992 to $2018^{*}$ (asterisk denotes data from January to October were obtained in 2018) 
Table 1 Summary of the studies on the nonvisual effects of the color temperature of light in the field of physiological anthropology published in the JPA from 1992 to 2018*

\begin{tabular}{|c|c|c|c|c|}
\hline \multirow[t]{2}{*}{ Author } & \multirow[t]{2}{*}{ Year } & \multicolumn{2}{|l|}{ Condition } & \multirow[t]{2}{*}{ Parameters Measured } \\
\hline & & Lighting & Others & \\
\hline \multirow[t]{2}{*}{ Deguchi T, Sato M. [13] } & \multirow[t]{2}{*}{1992} & Color temperature: $3000,5000,7500 \mathrm{~K}$ & & CNV \\
\hline & & Illuminance: 1000 Ix (fluorescent lamp) & & Reaction time \\
\hline \multirow[t]{3}{*}{ Kobayashi H, Sato M. [14] } & \multirow[t]{3}{*}{1992} & \multirow[t]{2}{*}{ Color temperature: $3000,5000,7500 \mathrm{~K}$} & \multirow[t]{3}{*}{ Addition task } & Blood pressure \\
\hline & & & & CFF \\
\hline & & $\begin{array}{l}\text { Illuminance: 320, 1000, } 2000 \text { Ix } \\
\text { (fluorescent lamp) }\end{array}$ & & $\begin{array}{l}\text { Accommodation } \\
\text { time of eye movement }\end{array}$ \\
\hline \multirow[t]{2}{*}{ Mukae H, Sato M. [15] } & \multirow[t]{2}{*}{1992} & Color temperature: $3000,5000,6700 \mathrm{~K}$ & \multirow[t]{10}{*}{ Addition task } & Heart rate \\
\hline & & $\begin{array}{l}\text { Illuminance: 100, 300, } 900 \mathrm{~lx} \\
\text { (fluorescent lamp) }\end{array}$ & & HRV \\
\hline \multirow[t]{4}{*}{ Morita T, Tokura H. [24] } & \multirow[t]{4}{*}{1996} & Color temperature: $3000,6500 \mathrm{~K}$ & & Rectal temperature \\
\hline & & Illuminance: 1000 Ix (fluorescent lamp) & & Melatonin \\
\hline & & \multicolumn{2}{|l|}{ Control: 50 lx (incandescent lamp) } & \\
\hline & & \multicolumn{2}{|l|}{ Exposure time: 21:00-02:00 } & \\
\hline \multirow[t]{4}{*}{ Noguchi H, Sakaguchi T. [27] } & \multirow[t]{4}{*}{1999} & Color temperature: $3000,5000 \mathrm{~K}$ & & HRV \\
\hline & & \multirow{3}{*}{$\begin{array}{l}\text { Illuminance: } 30,150 \text { Ix (fluorescent lamp) } \\
22 \text { min light exposure }+20 \text { min sleep in } \\
\text { darkness in the afternoon }\end{array}$} & & AAC \\
\hline & & & & EEG \\
\hline & & & & $\begin{array}{l}\text { Subjective evaluation } \\
\text { (drowsiness) }\end{array}$ \\
\hline \multirow[t]{3}{*}{$\begin{array}{l}\text { Yasukouchi A, Yasukouchi Y, } \\
\text { Ishibashi K. [28] }\end{array}$} & \multirow[t]{3}{*}{2000} & Color temperature: $3000,5000,7500 \mathrm{~K}$ & $\begin{array}{l}\text { Experiment 1: } \\
\text { Air Temp.: } 28^{\circ} \mathrm{C} \text { to } \\
18^{\circ} \mathrm{C} \text { during } 30 \mathrm{~min}\end{array}$ & Rectal temperature \\
\hline & & \multirow[t]{2}{*}{ Illuminance: 500 Ix (fluorescent lamp) } & \multirow{2}{*}{$\begin{array}{l}\text { Experiment 2: } \\
\text { Air Temp.: } 15^{\circ} \mathrm{C} \\
\text { for } 90 \mathrm{~min}\end{array}$} & Skin temperature \\
\hline & & & & $\begin{array}{l}\text { Metabolic heat } \\
\text { production }\end{array}$ \\
\hline Yasukouchi A, Ishibashi K. [31] & 2005 & $\begin{array}{l}\text { Review on nonvisual effects of color } \\
\text { temperature }\end{array}$ & & \\
\hline \multirow{4}{*}{$\begin{array}{l}\text { Kozaki T, Kitamura S, Higashihara Y, } \\
\text { Ishibashi K, Noguchi H, } \\
\text { Yasukouchi A. [25] }\end{array}$} & \multirow[t]{4}{*}{2005} & Color temperature: $3000,5000,6700 \mathrm{~K}$ & \multirow{4}{*}{$\begin{array}{l}\text { Sleep: } \\
\text { 02:00-09:00 (<10 Ix) }\end{array}$} & \multirow{4}{*}{$\begin{array}{r}\text { Polysomnogram } \\
(\mathrm{EEG}, \mathrm{EMG}, \mathrm{EOG})\end{array}$} \\
\hline & & Illuminance: 1000 Ix (fluorescent lamp) & & \\
\hline & & Exposure time: 19:30-02:00 & & \\
\hline & & Control: $2700 \mathrm{~K}$ (incandescent lamp) & & \\
\hline \multirow{7}{*}{$\begin{array}{l}\text { Katsuura T, Jin X, Baba Y, } \\
\text { Shimomura Y, Iwanaga K. [29] }\end{array}$} & 2005 & Experiment 1: & Experiment 1: & Experiment 1: \\
\hline & & Color temperature: $3000,7500 \mathrm{~K}$ & $\begin{array}{l}\text { Taste stimuli } \\
\text { (sweet, sour, bitter, salty) }\end{array}$ & $\begin{array}{l}\text { Taste threshold } \\
\text { (sweet, sour, bitter, salty) }\end{array}$ \\
\hline & & Illuminance: 200, 1500 Ix (fluorescent lamp) & & Saliva secretion \\
\hline & & Experiment 2: & & Experiment 2: \\
\hline & & Color temperature: $3000,5000,7000 \mathrm{~K}$ & & \\
\hline & & illuminance: $1000 \mathrm{~lx}$ & & \\
\hline & & $\begin{array}{l}\text { Exposure time: 00:00-01:00 during night } \\
\text { rest period }\end{array}$ & & \\
\hline
\end{tabular}


Table 1 Summary of the studies on the nonvisual effects of the color temperature of light in the field of physiological anthropology published in the JPA from 1992 to $2018^{*}$ (Continued)

\begin{tabular}{|c|c|c|c|c|}
\hline \multirow[t]{2}{*}{ Author } & \multirow[t]{2}{*}{ Year } & \multicolumn{2}{|l|}{ Condition } & \multirow[t]{2}{*}{ Parameters Measured } \\
\hline & & Lighting & Others & \\
\hline \multirow{3}{*}{$\begin{array}{l}\text { Ishibashi K, Kitamura S, Kozaki T, } \\
\text { Yasukouchi A. [26] }\end{array}$} & \multirow[t]{3}{*}{2007} & Color temperature: $3000,5000,6700 \mathrm{~K}$ & & Heart rate \\
\hline & & Illuminance: 1000 Ix (fluorescent lamp) & & HRV (FFT and CGSA) \\
\hline & & Exposure time: 19:30-02:00 & & \\
\hline \multirow{3}{*}{$\begin{array}{l}\text { Jin X, Katsuura T, Iwanaga K, } \\
\text { Shimomura Y, Inoue M. [30] }\end{array}$} & \multirow[t]{3}{*}{2007} & Color temperature: $3000,7500 \mathrm{~K}$ & \multirow{3}{*}{$\begin{array}{l}\text { Taste stimuli } \\
\text { (sweet, sour, bitter, salty) }\end{array}$} & \multirow[t]{3}{*}{ Electrogastrogram (EGG) } \\
\hline & & Illuminance: 200, 1500 Ix (fluorescent lamp) & & \\
\hline & & Exposure time: 14:25-ca.17:25 & & \\
\hline
\end{tabular}

*Data from January to October were obtained in 2018

color temperature and illuminance on taste threshold and brain functions. Our results suggested that the sensitivity to sweet and bitter tastes increased under high illuminance and tended to increase under high color temperature light exposure. We also found the alpha1 band ratio in EEG to be increased and beta band ratio to be decreased under low color temperature during a nighttime break [29]. The influence of color temperature and illuminance on autonomic nervous system (ANS) activity during taste stimulation was also examined using electrogastrography (EGG). This study reported the ratios of the normal wave component of the EGG during sweet and salty taste stimulation to be significantly higher than those observed during bitter taste stimulation, with significant increases of this ratio under low color temperature exposure [30]. Yasukouchi and Ishibashi [31] reviewed the nonvisual effects of color temperature based mainly on their studies with special reference to sleep architecture, arousal level, and ANS (including parameters such as HRV, blood pressure, and body temperature regulation).

\section{Nonvisual effects of the light intensity}

It has been well-known that the light intensity, which is expressed by illuminance, luminance, irradiance, etc., greatly affects the nonvisual responses [32]. This has also been a topic investigated in the field of physiological anthropology, with 20 papers published in the JPA over the years (Table 2). The vast majority of these papers inspected influences on the circadian rhythm [33-44]. A smaller number of papers have also addressed the influences of light intensity, when exposure occurs during a relatively short period, on the central nervous system (CNS) [45-47], the ANS as assessed by measurements such as HRV [46], PLR, nocturnal melatonin suppression [48], body temperature regulation [49], and serum tryptophan concentration and visuomotor and sensorimotor performance [50]. Two studies have specifically addressed effects on thermal regulation as measured by assessments of dressing behavior during cold exposure [51, 52].
Tokura and colleagues found bright light exposure during daytime to enhance evening fall and morning rise of body temperature when compared to dim light exposure. The acrophase of body temperature change also occurred earlier under bright light exposure [33]. Interestingly, diurnal bright light exposure was also found to activate some acute phase proteins, to increase nocturnal melatonin secretion, and to accelerate the fall in rectal temperature observed during the first half period of night sleep [34]. Importantly, bright light was also found to increase the time spent in bed, actual sleep time, and melatonin secretion in hospitalized elderly patients [35]. Diurnal bright light exposure also increased urine volume and creatinine clearance [39], but evening exposure to bright or dim light after bright light exposure in the daytime had no varying effect on digestion or absorption of dietary carbohydrates in the following morning's breakfast [40]. Kozaki et al. revealed the diurnal bright light exposure advanced the dim light melatonin onset [41]. Similarly, bright light exposure during the daytime was found to increased nocturnal melatonin secretion and to advance the phase of dim light melatonin onset [44]. Melatonin concentration during nocturnal light exposure after dim light exposure during daytime was decreased; however, exposure to bright light during the daytime blunted this response [43]. The combination of a tryptophan-rich breakfast and bright light exposure during daytime promoted melatonin secretion at night [42]. However, the intake of a tryptophan supplement during breakfast had no effect on nocturnal melatonin secretion [44].

Bright light exposure throughout nocturnal sleep deprivation suppressed the melatonin secretion and increase subjective ratings of sleepiness, and delayed the decline in heart rate and the increase in the theta and alpha wave activity [36, 38]. No significant correlation between individual differences in melatonin suppression by exposure to bright light during night and habitual bedtime in the 15 subjects in whom melatonin suppression occurred could be found [37]. Nevertheless, the habitual bedtime of the two subjects in whom melatonin 
Table 2 Summary of the studies on the nonvisual effects of the light intensity in the field of physiological anthropology published in the JPA from 1992 to 2018 (data from January to October were obtained in 2018)

\begin{tabular}{|c|c|c|c|c|}
\hline \multirow[t]{2}{*}{ Author } & \multirow[t]{2}{*}{ Year } & \multicolumn{2}{|l|}{ Condition } & \multirow[t]{2}{*}{ Parameters measured } \\
\hline & & Lighting & Others & \\
\hline \multirow{2}{*}{$\begin{array}{l}\text { Higuchi S, Watanuki S, } \\
\text { Yasukouchi A, Sato M. [45] }\end{array}$} & \multirow[t]{2}{*}{1997} & \multirow{2}{*}{$\begin{array}{l}\text { Luminance: } 10,100,320,1000, \\
1800 \mathrm{~cd} / \mathrm{m}^{2} \\
\text { Color temperature: } 2800 \mathrm{~K} \\
\text { (tungsten lamp with diffuser } \\
\text { and filters) }\end{array}$} & & CNV \\
\hline & & & & $\cdot E E G$ \\
\hline Park SJ, Tokura H. [33] & 1998 & $\begin{array}{l}\text { Illuminance: } 200,5000 \mathrm{Ix} \\
\text { (fluorescent lamp) } \\
\text { Exposure time: 06:30-19:30 }\end{array}$ & & Rectal temperature \\
\hline \multirow{2}{*}{$\begin{array}{l}\text { Noguchi H, Sakaguchi T, } \\
\text { Sato M. [46] }\end{array}$} & \multirow[t]{2}{*}{1999} & \multirow{2}{*}{$\begin{array}{l}\text { Illuminance: } 0.5,1,3,10,30 \mathrm{x} \\
\text { Color temperature: } 3000 \mathrm{~K} \\
\text { (fluorescent lamp) } \\
\text { Exposure time: } 2 \mathrm{~min}\end{array}$} & \multirow{2}{*}{$\begin{array}{l}\text { Subjects: young, elderly } \\
\text {.Time slots of experiment } \\
\text { 09:00-10:30, 10:30-12:00, } \\
\text { 13:00-14:30, 14:30-16:00, } \\
\text { 16:00-17:30 }\end{array}$} & $\cdot H R V \cdot E E G$ \\
\hline & & & & $\begin{array}{l}\text { Subjective evaluation } \\
\text { (discomfort) }\end{array}$ \\
\hline \multirow[t]{6}{*}{ Kim HE, Tokura H. [51] } & \multirow[t]{6}{*}{2000} & \multirow{6}{*}{$\begin{array}{l}\text { Illuminance: } 50,3000 \mathrm{~lx} \\
\text { (fluorescent lamp) } \\
\text { Exposure time: 09:30-14:30 }\end{array}$} & \multirow{6}{*}{$\begin{array}{l}\text { Air temp:: } 30 \text { to } 15^{\circ} \mathrm{C} \text { during } \\
\text { 15:00-17:00 } \\
\text { Elderly subjects }\end{array}$} & Rectal temperature \\
\hline & & & & Skin temperature \\
\hline & & & & Blood pressure \\
\hline & & & & Heart rate \\
\hline & & & & Dressing behavior \\
\hline & & & & $\begin{array}{l}\text { Subjective evaluation (temperature } \\
\text { sensation, thermal comfort) }\end{array}$ \\
\hline \multirow{8}{*}{$\begin{array}{l}\text { Kanikowska D, Hirata Y, } \\
\text { Hyun K, Tokura H. [34] }\end{array}$} & \multirow[t]{8}{*}{2001} & \multirow{8}{*}{$\begin{array}{l}\text { Illuminance: } 100,3000 \mathrm{~lx} \\
\text { (fluorescent lamp) } \\
\text { Exposure } \\
\text { time: } 07: 00-18: 00 \\
\text { first and } \\
\text { second days: } 100 \mathrm{~lx} \text {, third day } \\
3000 \mathrm{~lx}\end{array}$} & & Rectal temperature \\
\hline & & & & Melatonin \\
\hline & & & & C-reactive protein \\
\hline & & & & al-antichymotrypsin \\
\hline & & & & Transferrin \\
\hline & & & & a2-macroglobulin \\
\hline & & & & Haptoglobin \\
\hline & & & & Ceruloplasmin \\
\hline \multirow[t]{2}{*}{ Wakamura T, Tokura H. [35] } & \multirow[t]{2}{*}{2001} & \multirow{2}{*}{$\begin{array}{l}\text { Illuminance: } 3000 \mathrm{~lx} \\
\text { Color temperature: } 5000 \mathrm{~K} \\
\text { (fluorescent lamp) } \\
\text { Exposure time: 10:00-15:00 } \\
\text { Ambient light: } 50-300 \mathrm{~lx}\end{array}$} & \multirow{2}{*}{$\begin{array}{l}\text { Subjects, } 7 \text { hospitalized } \\
\text { elderly patients }\end{array}$} & Melatonin \\
\hline & & & & $\begin{array}{l}\text { Bed time, get up time, time in } \\
\text { bed, sleep start, sleep end, actual } \\
\text { sleep time, immobile mins }\end{array}$ \\
\hline \multirow{5}{*}{$\begin{array}{l}\text { Yokoi M, Aoki K, Shimomura Y, } \\
\text { Iwanaga K, Katsuura T. [36] }\end{array}$} & \multirow[t]{5}{*}{2003} & \multirow{5}{*}{$\begin{array}{l}\text { Illuminance: } 120,2800 \mathrm{~lx} \\
\text { (fluorescent lamp) } \\
\text { Exposure time: } 21: 00-04: 30 \\
\text { during sleep deprivation }\end{array}$} & \multirow{5}{*}{$\begin{array}{l}\text { Stroop color-word conflict } \\
\text { test }\end{array}$} & Melatonin \\
\hline & & & & Rectal temperature \\
\hline & & & & Skin temperature \\
\hline & & & & EEG \\
\hline & & & & Subjective sleepiness \\
\hline \multirow[t]{5}{*}{ Tokura H, Kim HE. [52] } & \multirow[t]{5}{*}{2005} & \multirow{5}{*}{$\begin{array}{l}\text { Illuminance: } 10,4000 \mathrm{Ix} \\
\text { Exposure time: } 10: 00-18: 00 \\
\text { Illuminance: } 10,3000 \mathrm{Ix} \\
\text { Exposure time: } 16: 00-23: 00\end{array}$} & \multirow{5}{*}{$\begin{array}{l}\text { Air temp.: } 30 \text { to } 15^{\circ} \mathrm{C} \text { during } \\
\text { 20:30-22:30 } \\
\text { Air temp.: } 30 \text { to } 15^{\circ} \mathrm{C} \text { during } \\
\text { 21:00-22:00 }\end{array}$} & Dressing behavior \\
\hline & & & & Rectal temperature \\
\hline & & & & Skin temperature \\
\hline & & & & Melatonin \\
\hline & & & & $\begin{array}{l}\text { Subjective evaluation (temperature } \\
\text { sensation, thermal comfort) }\end{array}$ \\
\hline
\end{tabular}


Table 2 Summary of the studies on the nonvisual effects of the light intensity in the field of physiological anthropology published in the JPA from 1992 to 2018 (data from January to October were obtained in 2018) (Continued)

\begin{tabular}{|c|c|c|c|c|}
\hline \multirow[t]{2}{*}{ Author } & \multirow[t]{2}{*}{ Year } & \multicolumn{2}{|l|}{ Condition } & \multirow[t]{2}{*}{ Parameters measured } \\
\hline & & Lighting & Others & \\
\hline \multirow{3}{*}{$\begin{array}{l}\text { Higuchi S, Motohashi Y, } \\
\text { Maeda T, Ishibashi K. [37] }\end{array}$} & \multirow[t]{3}{*}{2005} & \multirow{3}{*}{\multicolumn{2}{|c|}{$\begin{array}{l}\text { Illuminance: } 1000 \mathrm{~lx} \text { during } \\
2 \mathrm{~h} \text { from } 2 \mathrm{~h} \text { before peak } \\
\text { melatonin concentration }\end{array}$}} & Melatonin \\
\hline & & & & $\begin{array}{l}\text { Questionnaire (Morningness- } \\
\text { Eveningness Questionnaire) }\end{array}$ \\
\hline & & & & $\begin{array}{l}\text { Sleep diary (bedtime, rising time, } \\
\text { sleep quality, etc.) }\end{array}$ \\
\hline \multirow{6}{*}{$\begin{array}{l}\text { Yokoi M, Aoki K, Shimomura Y, } \\
\text { Iwanaga K, Katsuura T. [38] }\end{array}$} & \multirow[t]{6}{*}{2006} & \multirow{6}{*}{$\begin{array}{l}\text { Illuminance: } 120,2800 \mathrm{~lx} \\
\text { (fluorescent lamp) } \\
\text { Exposure time: } 21: 00-04: 30 \\
\text { during sleep deprivation }\end{array}$} & \multirow{6}{*}{$\begin{array}{l}\text { Stroop color-word conflict } \\
\text { test }\end{array}$} & Melatonin \\
\hline & & & & Rectal temperature \\
\hline & & & & Blood pressure \\
\hline & & & & Heart rate \\
\hline & & & & $\cdot H R V$ \\
\hline & & & & Task performance \\
\hline \multirow{2}{*}{$\begin{array}{l}\text { Hyun KJ, Nishimura S, } \\
\text { Tokura H. [39] }\end{array}$} & \multirow[t]{2}{*}{2006} & \multirow{2}{*}{\multicolumn{2}{|c|}{$\begin{array}{l}\text { Illuminance: } 80,5000 \mathrm{~lx} \\
\text { Second day: 07:00-23:00 in } 80 \mathrm{~lx} \\
\text { Third day: 07:00-15:00 in } 5000 \mathrm{~lx} \text {, } \\
\text { 15:00-23:00 in } 80 \mathrm{~lx}\end{array}$}} & Urine volume \\
\hline & & & & Creatinine clearance \\
\hline \multirow{4}{*}{$\begin{array}{l}\text { Yasukouchi A, Hazama T, } \\
\text { Kozaki T. [48] }\end{array}$} & \multirow[t]{4}{*}{2007} & \multirow{4}{*}{$\begin{array}{l}\text { PLR measurement: } \\
\text { Illuminance: } \\
\text { 1, 3, 30, } 600 \text { Ix } \\
\text { 5-min light exposure during } \\
\text { 01:00-02:30 } \\
\text { Melatonin measurement: } \\
\text { Illuminance: } 0,30,600 \text { Ix } \\
\text { Exposure time: 01:00-02:30 } \\
\text { Green (530 } \mathrm{nm} \text { ) (LED) }\end{array}$} & & PLR \\
\hline & & & & Melatonin \\
\hline & & & & Oral temperature \\
\hline & & & & Blood pressure \\
\hline Hirota N, Sone Y, Tokura H. [40] & 2010 & $\begin{array}{l}\text { Illuminance: } 2000 \mathrm{Ix} \\
\text { Exposure time: 07:00-15:00 } \\
\text { Illuminance: 50, 2000 Ix } \\
\text { Exposure time: 15:00-24:00 } \\
\text { (fluorescent lamp) }\end{array}$ & & $\begin{array}{l}\text { Breath hydrogen production } \\
\text { (unabsorbed dietary } \\
\text { carbohydrates) }\end{array}$ \\
\hline \multirow{4}{*}{$\begin{array}{l}\text { Kozaki T, Toda N, Noguchi H, } \\
\text { Yasukouchi A. [41] }\end{array}$} & \multirow[t]{4}{*}{2011} & \multirow{4}{*}{\multicolumn{2}{|c|}{$\begin{array}{l}\text { Illuminance: } 750,1500,3000 \text {, } \\
6000,12,000 \mathrm{Ix} \\
\text { Color temperature: } 5000 \mathrm{~K}\end{array}$}} & -Melatonin (DLMO) \\
\hline & & & & aMT6s concentration \\
\hline & & & & \\
\hline & & & & \\
\hline \multirow[t]{3}{*}{$\begin{array}{l}\text { Yoshinaga N, Fujita M, } \\
\text { Tanaka YL, Nemoto S. [47] }\end{array}$} & \multirow[t]{3}{*}{2011} & \multirow{3}{*}{$\begin{array}{l}\text { Illuminance: } 0,50,200 \mathrm{~lx} \\
\text { Same, lowering and raising } \\
\text { illuminance conditions } \\
\text { Exposure time: } 2 \mathrm{~min}+2 \mathrm{~min}\end{array}$} & \multirow[t]{3}{*}{$\begin{array}{l}\text { Time zone of experiment: } \\
\text { 11:00-17:00 }\end{array}$} & $\begin{array}{l}\text { Somatosensory evoked } \\
\text { potential (SEP) }\end{array}$ \\
\hline & & & & Subjective evaluation (pain) \\
\hline & & & & \\
\hline \multirow{5}{*}{$\begin{array}{l}\text { Kakitsuba N, Mekjavic IB, } \\
\text { Katsuura T. [49] }\end{array}$} & \multirow[t]{5}{*}{2011} & \multirow{5}{*}{$\begin{array}{l}\text { Illuminance: } \\
1050 \text { lx in winter } \\
510 \text { Ix in summer and winter } \\
\text { Color temperature: } 5000 \mathrm{~K} \\
\text { (fluorescent lamp) }\end{array}$} & \multirow{5}{*}{$\begin{array}{l}\text { Exercise: cycle ergometer } \\
\text { ( } 50 \% \text { maximum work) } \\
\text {-Maintained mean skin } \\
\text { temperature at } 28^{\circ} \mathrm{C} \text { by } \\
\text { perfusing a water-perfused } \\
\text { suit with water at } 25^{\circ} \mathrm{C} .\end{array}$} & Core interthreshold zone (CIZ) \\
\hline & & & & Rectal temperature \\
\hline & & & & Skin temperature \\
\hline & & & & Sweat rate \\
\hline & & & & Oxygen uptake \\
\hline
\end{tabular}


Table 2 Summary of the studies on the nonvisual effects of the light intensity in the field of physiological anthropology published in the JPA from 1992 to 2018 (data from January to October were obtained in 2018) (Continued)

\begin{tabular}{|c|c|c|c|c|}
\hline \multirow[t]{2}{*}{ Author } & \multirow[t]{2}{*}{ Year } & \multicolumn{2}{|l|}{ Condition } & \multirow[t]{2}{*}{ Parameters measured } \\
\hline & & Lighting & Others & \\
\hline \multirow{3}{*}{$\begin{array}{l}\text { Fukushige H, Fukuda Y, Tanaka M, } \\
\text { Inami K, Wada K, Tsumura Y, } \\
\text { Kondo M, Harada T, Wakamura T, } \\
\text { Morita T. [42] }\end{array}$} & \multirow{3}{*}{2014} & \multirow{3}{*}{$\begin{array}{l}\text { Illuminance: > } 5000 \text { lx (Bright), } \\
<50 \text { lx (Dim) } \\
\text { Exposure time: 07:30-18:00 }\end{array}$} & \multirow{3}{*}{$\begin{array}{l}\text { Tryptophan intake: } \\
\text { Rich } \\
476 \text { mg } \\
\text { Poor } 55 \text { mg } \\
\text { Poor*dim, Poor*bright, } \\
\text { Rich*dim, Rich*bright }\end{array}$} & Melatonin (DLMO) \\
\hline & & & & $\begin{array}{l}\text { Activity (sleep efficiency, sleep } \\
\text { latency) }\end{array}$ \\
\hline & & & & $\begin{array}{l}\text { Subjective estimation (sleepiness, } \\
\text { sleep maintenance, worries, } \\
\text { integrated sleep feeling, sleep } \\
\text { initiation) }\end{array}$ \\
\hline
\end{tabular}

Kozaki T, Kubokawa A, Taketomi R, Hatae K. [43]
Nagashima S, Yamashita M Tojo C, Kondo M, Morita T, Wakamura T. [44]

Schobersberger W, Blank C, Hanser F, Griesmacher A, Canazei M, Leichtfried V. [50]

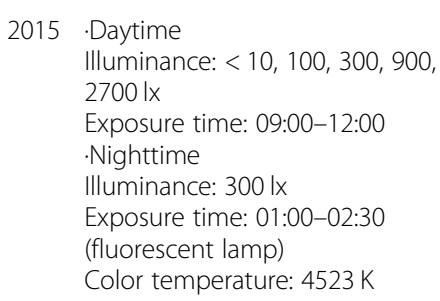

Tryptophan (TRP) intake at breakfast

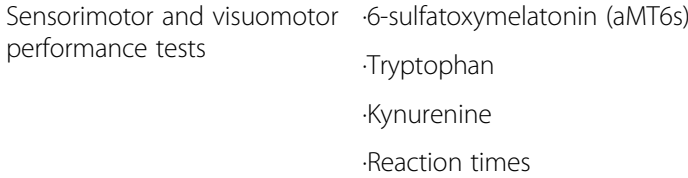

LED light-emitting diode

suppression did not occur was earlier than that of the other subjects [37].

The following studies examined the influence of light intensity when exposure occurs during a relatively short period of time. The effects of luminance on CNV and spontaneous EEG during $10 \mathrm{~min}$ of simple reaction tasks were measured, and an inverted- $U$ shape relationship between the changes in arousal level by light stimulation and resultant CNV was identified [45]. Noguchi et al. [46] studied the effects of exposure to different illuminances $(0.5,1,3,10,30 \mathrm{~lx})$ during $2 \mathrm{~min}$ after dark adaptation and found the LF/(LF + HF) of the HRV to follow a V-shaped trend, with a minimum on the 3 -lx condition. The proportion of alpha wave activity on EEG fell markedly on $3 \mathrm{~lx}$ or higher illuminance conditions [46]. The effects of lowering or raising illuminance on somatosensory evoked potential (SEP) and subjective sensory evaluation were also examined. It was found that the SEP amplitude and the subjective sensory evaluation tended to decrease when illuminance was lowered and tended to increase when illuminance was raised [47]. The relationship between individual differences in nocturnal melatonin suppression induced by lighting and the individual differences on PLR were also investigated. Interestingly, it was found that subjects whose melatonin secretions were easily suppressed typically presented low PLR [48]. Kakitsuba et al. [49] examined the seasonal difference in the core interthreshold zone (CIZ)-defined as the range between core temperature at the onset of shivering and that at the onset of sweating-during winter and summer, in two different light exposure conditions. They found CIZ in summer to be higher than that observed during winter in both light exposure conditions. The effect of the morning bright light (07:40-08:10, $5000 \mathrm{~lx})$ exposure on sensorimotor and visuomotor performance and serum tryptophan and its metabolites was evaluated [50]. These data suggest that morning bright light exposure has a limited effect on visuomotor and sensorimotor performance, but can potentially alter serum tryptophan concentration.

Tokura and colleagues [51] examined the effects of exposure to bright or dim light on dressing behavior and thermoregulatory responses in elderly people during exposure to cold in the afternoon (decreases from 30 to $15^{\circ} \mathrm{C}$ ). These authors found that the subjects tended to feel cooler and dressed more quickly with thicker clothes after dim light, when compared with 
Table 3 Summary of the studies on the nonvisual effects of monochromatic light in the field of physiological anthropology published in the JPA from 1992 to 2018 (data from January to October were obtained in 2018)

\begin{tabular}{|c|c|c|c|c|}
\hline \multirow[t]{2}{*}{ Author } & \multirow[t]{2}{*}{ Year } & \multicolumn{2}{|l|}{ Condition } & \multirow[t]{2}{*}{ Parameters measured } \\
\hline & & Lighting & Others & \\
\hline \multirow{4}{*}{$\begin{array}{l}\text { Morita T, Tokura H, } \\
\text { Wakamura T, Park SJ, } \\
\text { Teramoto Y. [53] }\end{array}$} & \multirow[t]{4}{*}{1997} & \multirow{4}{*}{\multicolumn{2}{|c|}{$\begin{array}{l}\text { Blue }(435 \mathrm{~nm}) \text {, green }(545 \mathrm{~nm}) \text {, red } \\
(610 \mathrm{~nm}) \\
\text { Illuminance: } 1000,2500 \mathrm{~lx} \\
\text { Control } 50 \text { lx (incandescent lamp) } \\
\text { Exposure time: 04:00-09:00 }\end{array}$}} & Rectal temperature \\
\hline & & & & Melatonin \\
\hline & & & & \\
\hline & & & & \\
\hline Morita T, Tokura H. [54] & 1998 & \multicolumn{2}{|l|}{$\begin{array}{l}\text { Review on the influence of different } \\
\text { wavelengths of light }\end{array}$} & $\begin{array}{l}\text { Rectal temperature } \\
\text {-Melatonin }\end{array}$ \\
\hline \multirow{3}{*}{$\begin{array}{l}\text { Katsuura T, Yasuda T, } \\
\text { Shimomura Y, } \\
\text { Iwanaga K. [67] }\end{array}$} & \multirow[t]{3}{*}{2007} & \multirow{3}{*}{$\begin{array}{l}\text { Red, blue } \\
\text { Illuminance: } 310 \text { lx } \\
\text { (fluorescent lamp) }\end{array}$} & \multirow{3}{*}{$\begin{array}{l}\text { Time zone of experiment: } \\
\text { 10:00-18:00 }\end{array}$} & Time production (90 s, 180 s) \\
\hline & & & & Event-related potential (P300) \\
\hline & & & & $\begin{array}{l}\text { Subjective evaluation (sleepiness, } \\
\text { tension, fatigue, comfort, } \\
\text { concentration) }\end{array}$ \\
\hline \multirow{4}{*}{$\begin{array}{l}\text { Yoto A, Katsuura T, } \\
\text { Iwanaga K, Shimomura Y. } \\
{[70]}\end{array}$} & \multirow[t]{4}{*}{2007} & \multirow{4}{*}{$\begin{array}{l}\text { Red, green, blue (sheets of paper in } \\
\text { A2 size) } \\
\text { Illuminance at surface of the color } \\
\text { paper: } 500 \text { lx }\end{array}$} & \multirow{4}{*}{$\begin{array}{l}\text { Time zone of experiment: } \\
\text { 10:00-17:00 }\end{array}$} & $\cdot E E G$ \\
\hline & & & & $\cdot A A C$ \\
\hline & & & & Blood pressure \\
\hline & & & & $\begin{array}{l}\text { Subjective evaluation (lightness vs. } \\
\text { heaviness, calm vs. excited, sleepy } \\
\text { vs. not sleepy, cold vs. warm, etc.) }\end{array}$ \\
\hline \multirow{3}{*}{$\begin{array}{l}\text { An M, Huang J, } \\
\text { Shimomura Y, Katsuura T. } \\
\text { [55] }\end{array}$} & \multirow[t]{3}{*}{2009} & \multirow{3}{*}{\multicolumn{2}{|c|}{$\begin{array}{l}\text { Blue }(458 \mathrm{~nm}) \text {, green }(550 \mathrm{~nm}) \\
\text { Irradiance: } 9.8 \mu \mathrm{W} / \mathrm{cm}^{2} \\
\text { (halogen lamp with interference filter) } \\
\text { Exposure time: daytime and nighttime }\end{array}$}} & Event-related potential (P300) \\
\hline & & & & $\cdot A A C$ \\
\hline & & & & Subjective evaluation (sleepiness) \\
\hline
\end{tabular}
(ca. $9.25 \mathrm{~h}$ before the respective averaged

Higuchi S, Fukuda T, Miura N. [56] Kozaki T, Takahashi M,

2011 Light source: ceiling light (500 lx)

Color temperature: $4200 \mathrm{~K}$

(fluorescent lamp)

nonvisor cap (500 lx)

red-visor cap (ca. 160 lx)

blue-visor cap (ca. 160 lx)

Exposure time: 23:00-03:00

Fukuda Y, Higuchi S,

Yasukouchi A, Morita T. [68]

Katsuura T, Ochiai $Y$, Senoo T, Lee S, Takahashi

Y, Shimomura Y. [58]

Kakitsuba N, Mekjavic IB, Katsuura T. [69]
2012 468, 508, 593, 633 nm (LED) Masking-cone stimuli: $2000 \mathrm{~ms}(20 \mathrm{~Hz})$

Test stimulus (mRGC or cone): $250 \mathrm{~ms}$

2012 Blue (471, $467 \mathrm{~nm})$, white (color temperature $3034 \mathrm{~K}$ ) Irradiance: $12 \mu \mathrm{W} / \mathrm{cm}^{2}$ (LED)

2013 Blue $(436 \mathrm{~nm})$, red $(612 \mathrm{~nm})$ Illuminance: 500, 1000 lx White (color temperature $5000 \mathrm{~K}$ ) Illuminance: 1000 lx (fluorescent lamp)
Psychomotor vigilance task (PVT)

-Melatonin-Reaction time, no. of lapses (PVT).Subjective evaluations (sleepiness, brightness)

-Electroretinogram (ERG)

Pupillary constriction

-EEG

Subjective evaluation (bluish, sleepiness)

Exercise: cycle ergometer (50\% maximum work) -Maintained mean skin temperature at $28^{\circ} \mathrm{C}$ by perfusing a water-perfused suit with water at $25^{\circ} \mathrm{C}$.

Summer and winter

-Time zone of experiment 11:00-15:00

Lee SI, Hida A,

Tsujimura S, Morita T,

Mishima K, Higuchi S. [59]

2013 Blue (465 nm), green (536 nm), red (632 nm) Intensity: 12, 13, 14, 14.5 and $15 \mathrm{log}$ photons $/ \mathrm{cm}^{2} \mathrm{~s}$ (LED)
Time zone of experiment: 10:00-17:00
Core interthreshold zone (ClZ) -Rectal temperature

Skin temperature Sweat rate Oxygen uptake
-Pupil size -Melanopsin gene of single nucleotide polymorphism of rs1079610 (I394T):TT, TC, CC 
Table 3 Summary of the studies on the nonvisual effects of monochromatic light in the field of physiological anthropology published in the JPA from 1992 to 2018 (data from January to October were obtained in 2018) (Continued)

\begin{tabular}{|c|c|c|c|c|}
\hline \multirow[t]{2}{*}{ Author } & \multirow[t]{2}{*}{ Year } & \multicolumn{2}{|l|}{ Condition } & \multirow[t]{2}{*}{ Parameters measured } \\
\hline & & Lighting & Others & \\
\hline $\begin{array}{l}\text { Lee S, Ishibashi S, } \\
\text { Shimomura Y, } \\
\text { Katsuura T. [60] }\end{array}$ & 2016 & $\begin{array}{l}\text { Blue }(470 \mathrm{~nm}) \text {, green }(532 \mathrm{~nm}) \\
\text { Irradiance: } 10,15,20 \mu \mathrm{W} / \mathrm{cm}^{2} \\
\text { Pulse width: } 1 \mathrm{~ms}(\mathrm{LED}) \\
\text { Blue, green, blue + green }\end{array}$ & $\begin{array}{l}\text { Time zone of experiment: } \\
\text { 09:00-12:00, 13:00-16:00 }\end{array}$ & $\begin{array}{l}\text { Pupillary constriction } \\
\text { Subjective evaluation (sleepiness) }\end{array}$ \\
\hline $\begin{array}{l}\text { Yuda E, Ogasawara H, } \\
\text { Yoshida Y, Hayano J. [64] }\end{array}$ & 2016 & $\begin{array}{l}\text { Blue }(10,5,2 \mid x) \\
\text { Green }(71 \mid x) \\
\text { Red }(39 \mid x) \\
\text { (OLED) }\end{array}$ & $\begin{array}{l}\text { Time zone of experiment: } \\
\text { 08:30-13:00 }\end{array}$ & Heart rate.HRV \\
\hline $\begin{array}{l}\text { Yuda E, Ogasawara H, } \\
\text { Yoshida Y, Hayano J. [65] }\end{array}$ & 2017 & $\begin{array}{l}\text { Blue }(483 \mathrm{~nm}, 13 \mathrm{~lx}) \\
\text { Green }(555 \mathrm{~nm}, 91 \mathrm{~lx}) \\
\text { White }(158 \mathrm{|x}) \\
\text { (OLED) } \\
\text { Exposure time: } 10 \mathrm{~min}+5 \mathrm{~min} \\
\text { PVT under white fluorescent light } \\
\text { (illuminance, } 300 \mathrm{~lx} \text { ) }\end{array}$ & $\begin{array}{l}\text { Psychomotor vigilance } \\
\text { test (PVT) } \\
\text { Time zone of experiment: } \\
\text { 09:30-14:00 }\end{array}$ & $\begin{array}{l}\text { Heart rate.HRV.Reaction } \\
\text { time, minor lapse }(\mathrm{PVT})\end{array}$ \\
\hline $\begin{array}{l}\text { Dai Q, Uchiyama Y, } \\
\text { Lee S, Shimomura Y, } \\
\text { Katsuura T. [61] }\end{array}$ & 2017 & $\begin{array}{l}\text { Blue }(467 \mathrm{~nm}) \\
\text { Irradiance: } 7.5,15,30 \mu \mathrm{W} / \mathrm{cm}^{2} \\
\text { Pulse width: } 50,100,200 \mu \mathrm{s} \\
\text { White (color temperature } \\
\left.2878 \mathrm{~K}, 14.75 \mu \mathrm{W} / \mathrm{cm}^{2}\right)(\mathrm{LED}) \\
\text { Exposure time: } 12 \mathrm{~min}\end{array}$ & & $\begin{array}{l}\text { Pupillary constriction } \\
\text { EEG } \\
\text { Subjective } \\
\text { evaluation (concentration, sleepiness, } \\
\text { perception of blueness) }\end{array}$ \\
\hline $\begin{array}{l}\text { Yuda E, Ogasawara H, } \\
\text { Yoshida Y, Hayano J. [66] }\end{array}$ & 2017 & $\begin{array}{l}\text { Blue }\left(485 \mathrm{~nm}, 8.02 \mu \mathrm{W} / \mathrm{cm}^{2}\right) \\
\text { Orange }\left(622 \mathrm{~nm}, 6.54 \mu \mathrm{W} / \mathrm{cm}^{2}\right) \\
\text { (OLED) } \\
\text { Exposure time: } 30 \mathrm{~min} \\
\text { during lunch break } \\
5 \mathrm{~min} \text { of PVT under white fluorescent } \\
\text { light (color temperature } 4010 \mathrm{~K} \text {, } \\
\text { illuminance } 450 \mathrm{Ix} \text { ) }\end{array}$ & $\begin{array}{l}\text { Psychomotor vigilance } \\
\text { test (PVT) }\end{array}$ & $\begin{array}{l}\text { Heart rate } \\
\text { HRV } \\
\text {.Reaction time, minor } \\
\text { lapse (PVT) }\end{array}$ \\
\hline $\begin{array}{l}\text { Lee S, Muto N, } \\
\text { Shimomura } Y, \\
\text { Katsuura T. [62] }\end{array}$ & 2017 & $\begin{array}{l}\text { Blue }(466 \mathrm{~nm}) \\
\text { Green }(527 \mathrm{~nm}) \\
\text { Irradiance: } 20 \mu \mathrm{W} / \mathrm{cm}^{2} \\
\text { Pulse width: } 1 \mathrm{~ms} \\
\text { Inter-stimulus interval: } 0,250 \text {, } \\
500,750,1000 \mathrm{~ms}\end{array}$ & & Pupillary constriction \\
\hline $\begin{array}{l}\text { Lee } S \text {, Uchiyama Y, } \\
\text { Shimomura Y, } \\
\text { Katsuura T. [63] }\end{array}$ & 2017 & $\begin{array}{l}\text { Blue }(464 \mathrm{~nm}) \\
\text { Green }(526 \mathrm{~nm}) \\
\text { Pulse width: } 2.5 \mathrm{~ms} \\
\text { Photon density: } \\
15.2 \text { log photons } / \mathrm{cm}^{2} / \mathrm{s} \\
\text { Blue, green, blue }+ \text { green }\end{array}$ & & $\begin{array}{l}\text { Electroretinogram (ERG) } \\
\text { EEG } \\
\text { Visual } \\
\text { evoked potential } \\
\text {.Pupillary constriction } \\
\text {-Subjective evaluations } \\
\text { (bluish, greenish) }\end{array}$ \\
\hline $\begin{array}{l}\text { Kozaki T, Hidaka Y, } \\
\text { Takakura JY, } \\
\text { Kusano Y. [57] }\end{array}$ & 2018 & $\begin{array}{l}\text { Blue }(465 \mathrm{~nm}) \\
\text { Irradiance: } 52 \mu \mathrm{W} / \mathrm{cm}^{2} \\
\text { 100-Hz flickering ( } 10 \% \text { duty tatio) } \\
\text { non-flickering light } \\
\text { (LED) } \\
\text { Dim (<3 Ix) } \\
\text { Exposure time: } 01: 00-02: 30\end{array}$ & & Melatonin \\
\hline
\end{tabular}


bright light exposure. They also reviewed their own works about the effects of bright light exposure on dressing behavior in the cold and discussed the underlying physiological mechanisms [52].

\section{Nonvisual effects of monochromatic light}

Studies on this specific topic have contributed with a significant number of papers (Table 3). The number of papers addressing this question has increased over the last years. Papers have mainly focused on the effects of monochromatic light on circadian rhythm [53-57] and pupillary constriction [58-63]. A few other papers have studied on HRV [64-66], time sense and respective eventrelated potentials [67], electroretinogram (ERG) [68], and body temperature regulation [69]. One paper has specifically focused on the influence of the colored paper [70].

We now discuss the studies on the effects of monochromatic light on circadian rhythm. Morita and Tokura et al. investigated the effects of exposure to blue, green, and red bright lights from 04:00 to 09:00 on the rectal temperature and melatonin secretion. They found that green light tend to promote an increase of rectal temperature and enhance the fall of melatonin secretion when compared to control dim and red lights [53]. The same authors also reviewed the papers on the influence of monochromatic light on human biological rhythms. In this review, the authors suggest that the effects of light on core temperature and melatonin secretion may vary depending on its wavelength, and hypothesize that the photoreceptors putatively responsible for transmitting light information that affects the biological rhythms are the M-cones [54]. In another study, authors examined the time-of-day-dependent effects of blue and green lights on human cognitive function. Blue light exposure caused a significantly larger P300 amplitude than that observed with green light, and the P300 amplitude at nighttime during blue light exposure was also larger than that occurred with the same color at daytime [55]. Higuchi et al. [56] studied the effects of a red-visor cap, which could cut the short wavelengths of light received from the upper visual field, on light-induced melatonin suppression, performance, and sleepiness during the night. They found the red-visor cap to be effective in preventing melatonin suppression, with no adverse effects on vigilance performance, brightness, or visibility. Recently, Kozaki et al. [57] evaluated light-induced melatonin suppression under dim, $100-\mathrm{Hz}$ flickering and non-flickering blue light conditions at night (01:00-02:30). They found that melatonin concentration was significantly lower under non-flickering light than under dim light $15 \mathrm{~min}$ after the light exposure, whereas after 01:30, the mean melatonin concentrations were significantly lower under both $100-\mathrm{Hz}$ flickering and non-flickering blue light than under dim light. These findings suggest that $100-\mathrm{Hz}$ flickering light may have the same effect on melatonin secretion as non-flickering light.

The effects of monochromatic light on the pupillary constriction or PLR were evaluated in several studies. We have measured the pupil diameter, EEG responses, and the subjective bluish score during four lighting conditions, including a blue pulsed-light condition of $100-\mu \mathrm{s}$ pulse width with a $10 \%$ duty ratio. Interestingly, we found that the pupillary constriction in the blue pulsed-light condition was greater than that observed in the steady blue light condition. Importantly, both conditions had identical melanopsin-stimulating irradiance [58]. We then examined the effects of separate and simultaneous exposure to extremely short pulses of blue and green lights at different irradiance levels on pupillary constriction. We found that pupillary constriction during the simultaneous exposure to blue and green lights was decreased when compared with separate exposure to blue light, despite the double irradiance intensity of the combination. These findings indicate that the effect of blue light on ipRGCs may be inhibited by simultaneous exposure to green light [60]. In another study, the effects of blue pulsed light with different combination of irradiance intensities and pulse widths in equal quantities (product of irradiance and pulse width) were tested for synergistic effects with white pulsed light. Pupillary constriction was found to be greater under high irradiance with short pulse width and under middle irradiance with middle pulse width, when compared with low irradiance with long pulse width condition. These findings indicate that higher intensity with shorter pulsed width of blue pulsed light produces a more significant influence on ipRGCs, even when the quantity of blue pulsed light is held constant [61]. The effects of both simultaneous and successive exposure to blue and/or green pulsed light on PLR were investigated using extremely short pulses $(1 \mathrm{~ms})$ of blue and green lights with inter-stimulus intervals (ISIs) ranging from 0 to $1000 \mathrm{~ms}$. It was found that successive irradiation with pulses of blue and green lights at ISIs $\geq 500 \mathrm{~ms}$ induced pronounced pupillary constriction [62]. We also evaluated the effects of extremely short blue and green pulsed lights on visual evoked potential, pupillary constriction, ERG, and subjective evaluations. We found that pupillary constriction during the simultaneous exposure to blue and green pulsed lights was significantly lower than that observed during the blue pulsed light exposure, despite the double irradiance intensity of the combination. We also found the $b /|a|$ wave of ERG during the simultaneous exposure to blue and green pulsed lights to be lower than that observed during the blue pulsed light exposure [63]. The association between melanopsin gene polymorphism (I394T) and pupillary light reflex under diverse photic conditions, including different intensities $\left(12,13,14,14.5\right.$, and $15 \log$ photons $\left./ \mathrm{cm}^{2} / \mathrm{s}\right)$ and wavelengths (465, 536 and $632 \mathrm{~nm}$ ), was studied [59]. 
Table 4 Summary of other studies on the nonvisual effects of light in the field of physiological anthropology published in the JPA from 1992 to 2018 (data from January to October were obtained in 2018)

\begin{tabular}{lll}
\hline Author & Year & Condition \\
\cline { 3 - 3 } & Küller R. [78] & Lighting \\
\hline Yasukouchi A. [79] & $\begin{array}{l}\text { Review on the influence of light } \\
\text { on circadian rhythms and } \\
\text { circannual rhythms }\end{array}$ \\
& $2005 \begin{array}{l}\text { Review on human adaptability to } \\
\text { artificial light environment, based } \\
\text { on evaluations from CNS, ANS, and } \\
\text { biological rhythm. }\end{array}$ \\
&
\end{tabular}

Tsumura Y, Hirota N, Tokura H, 2005

Sone $Y$, Lesinski F, Rutkowska D,

Barinow-Wojewodzki A. [71]

Hanifin JP, Brainard GC. [80]

Ueno-Towatari T, Norimatsu $\mathrm{K}$, Blazejczyk K, Tokura H,

Morita T. [72]

Nakade $\mathrm{M}$, Takeuchi $\mathrm{H}$,

Taniwaki N, Noji T,

Harada T. [73]

Tanaka M, Hosoe K

Hamada T, Morita T. [75]

Nakade M, Akimitsu O,

Wada K, Krejci M, Noji T,

Taniwaki N, Takeuchi H,

Harada T. [74]

Crowley SK

Youngstedt SD. [81]

Lee SI, Hida A, Kitamura S,

Mishima K, Higuchi S. [76]

Daneault $\mathrm{V}$, Dumont $\mathrm{M}$

Masse $\mathrm{E}$, Vandewalle $\mathrm{G}$

Carrier J. [82]
Subjects: Japanese and Polish

-Tests were conducted at the summer

of 2004 in Japan and Poland.

Others

Parameters measured

Orocecal transit time (OOCT)

for minestrone and for

lactosucrose

amount of undigested

carbohydrate of minestrone

(UCM)

2007 Review on photoreception for circadian, neuroendocrine, and neurobehavioral regulation.

2007 Field study

Subjects: infants ( $2-6$ years)

Subjects: elder males and females studied before and after their cataract surgery
Melatonin (every $3 \mathrm{~h}$ from 10:00 on Thursday to 07:00 on Friday in four seasons) -Light intensity (every 1 min for 5 day in four seasons)

-Integrated questionnaire (Morningness-Eveningness Questionnaire, sleep habits, breakfast habits, mental health, morning exposure to sunlight)

Melatonin.Amounts of light exposure

-Activity (sleep efficiency, sleep latency) Wake-up time, retiring time

Integrated questionnaire (Morningness-Eveningness Questionnaire, sleep habits, breakfast habits, mental health, morning exposure to sunlight)
2012 Review on light therapy for perinatal depression

2014

2016 Review on neural pathways underlying effects of light on NIF functions, and discussed eye and cerebral mechanisms associated with aging
- Melanopsin gene of single nucleotide polymorphism of Ile394Thr:TT, TC, CC Morningness-Eveningness Questionnaire sleep habits (bedtime, wake time, sleep duration) 
Table 4 Summary of other studies on the nonvisual effects of light in the field of physiological anthropology published in the JPA from 1992 to 2018 (data from January to October were obtained in 2018) (Continued)

\begin{tabular}{lllll}
\hline Author & Year & Condition & Parameters measured \\
\cline { 3 - 4 } & & Lighting & Others & Melatonin \\
\hline Adamsson M, Laike T, & 2017 & Field study & South of Sweden $\left(56^{\circ} \mathrm{N}\right)$ & Cortisol \\
Morita T. [77] & & February 2008 to January 2009 & -Light exposure (illuminance) \\
& & 3-day/month, beginning at 12:00 on & Spectral composition of the \\
& & Tuesday and & exposing irradiance \\
\hline
\end{tabular}

They found that the pupil sizes of the TC $+\mathrm{CC}$ genotypes were significantly smaller than those of the TT genotype under a blue $(463 \mathrm{~nm})$ light condition with $15 \mathrm{log}$ photons $/ \mathrm{cm}^{2} / \mathrm{s}$, and the relative pupil constrictions of the $\mathrm{TC}+\mathrm{CC}$ genotypes were greater than those of the TT genotype under both blue and green conditions with high intensities. These findings suggest that the melanopsin gene polymorphism (I394T) functionally interacts with pupillary light reflex, depending on light intensity and, particularly, wavelength [59].

The effects of monochromatic light on ANS, as assessed by HRV measurements, were studied in several works. Yuda et al. [64] recorded electrocardiogram activity during a 3-min darkness and a 6-min exposure to a blue, green, or red light. They found a greater decrease in HF power for blue light when compared to red and green lights. The decreases in HF power even lasted during the period of darkness that followed exposure. They analyzed HRV during blue, green and white light exposure and reported an increase in heart rate and a decrease in HF during blue light exposure, when compared with green and white lights [65]. In the same study, evaluating performance during a psychomotor vigilance test after exposure revealed a decrease in the number of minor lapses and in the variation of reaction times, after exposure to blue light when compared to the green light [65]. The same authors also compared the effects of blue and orange lights on HRV and behavioral alertness during the lunch break. They observed that blue light, when compared with orange light, enhanced autonomic arousal. This effect was not sustained and was not accompanied by changes in behavioral reports of alertness after exposure [66].

The effects of monochromatic light have been studied from other viewpoints as well. We examined effects of blue and red lights on time sense and correspondent event-related potentials and revealed that the 180-s time interval produced in the red light condition was shorter and the peak latency of P300 in the red light was also shorter than that in the blue light [67]. Fukuda et al. [68] compared the waveform of ERG by using the illumination system, which modulated stimulus levels to the ipRGCs and cones independently. They found that the response to the ipRGC stimulus was significantly higher than that observed to the cone stimulus, at approximately $80 \mathrm{~ms}$ after the onset of the stimuli, and tended also to be higher than that observed to the cone stimulus, at approximately $280 \mathrm{~ms}$ after the stimuli. CIZs during exposure to blue and red lights at 500 and $1000 \mathrm{~lx}$ in both summer and winter were compared in another study. The authors reported on a significant difference in the CIZs between red and blue lights at $1000 \mathrm{~lx}$ in the winter. Significant seasonal differences under red and blue lights were also observed at $1000 \mathrm{~lx}$ [69]. The effects of colored paper (blue, green, and red) on EEG, blood pressure, and subjective evaluations were investigated. It was found that $A A C$ values were higher while looking at blue paper when compared to red. Alpha band power of EEG for red and green was also higher than that observed for blue [70].

\section{Other researches on nonvisual effects of light}

In this section, we discuss the papers categorized as others, as previously described (Table 4). Tsumura et al. [71] compared the efficiency of carbohydrate absorption, as assessed by the breath hydrogen test, between Japan and Poland habitants. They found orocecal transit times for lactosucrose and the amount of undigested carbohydrate of minestrone of Japanese subjects to be significantly longer and larger, respectively, than those of Polish. The authors hypothesized that longer daytime and dimmer night light conditions in Poland could have affected morning digestive activity of Polish subjects, which presented enhanced absorption of dietary carbohydrate in the morning meal. Seasonal variations in melatonin secretion and surrounding light conditions were examined in another study. It was detected that peak values of melatonin secretion were higher in autumn than in other seasons. They also found that the relationship between peak level of melatonin secretion and the amount of daytime light exposure to more than $1000 \mathrm{~lx}$ was significant only in the autumn [72]. Nakade et al. [73] investigated the effects of tryptophan intake at breakfast and morning exposure to sunlight by using an integrated questionnaire in Japanese infants. They found that infants exposed to sunlight for 30-60 min in the morning showed distinctive shifting effects to morning type with protein intake, when compared to those exposed to sunlight for less than $20 \mathrm{~min}$. The same 
authors also examined potential synergistic effects between tryptophan and vitamin B6 intake and morning exposure to sunlight in the same population. They found positive correlations between the Morningness-Eveningness (M-E) score and the amount of tryptophan intake and also between the M-E score and the vitamin B6 intake [74]. The positive correlation between M-E score and amount of tryptophan intake was only significant for infants who were exposed to sunlight for longer than $10 \mathrm{~min}$ after breakfast [74]. Melatonin secretion profiles and sleep patterns before and after cataract surgery were investigated in elderly patients. No significant differences could be identified in melatonin secretion, sleep parameters, or sleepiness before and after the surgery; however, the sleep efficiency of subjects with earlier wake-up and retiring times was higher than that observed in subjects with later wake-up and retiring times [75]. Lee et al. [76] analyzed the association between genotype of one polymorphism in the melanopsin gene (OPN4*Ile394Thr) and sleep/wake timing. They found that the sleep/wake timing of subjects with the CC genotype was significantly later than that of subjects with TT or TC genotypes. Adamsson et al. [77] examined the natural pattern of diurnal and seasonal light exposure and the seasonal variations in the circadian change of melatonin and cortisol concentrations in a group of Swedish office workers. They found large seasonal differences in the daily amount of light exposure across the year, alongside with a seasonal variation in melatonin concentration which presented a larger peak during the winter.

Küller [78] reviewed the influence of light on circadian and circannual rhythms in 2002. In this review, he discussed topics such as shift work, jet lag, and seasonal affective disorder and referred to the effectiveness of bright light exposure to alleviate these problems. Yasukouchi [79] discussed the physio-anthropological approach to evaluate human adaptability to artificial light environment in a review article published in 2005. He summarized the studies on the light environment in the field of physiological anthropology. Hanifin and Brainard [80] elaborated on the history of the study of action spectrum effects on circadian, neuroendocrine, and neurobehavioral responses. This study also included mentions to the discovery of melanopsin and ipRGCs and also discussed the use of light therapies for clinical and nonclinical applications. Crowley and Youngstedt [81] discussed the rationale behind the use of bright light therapy for perinatal depression and the available evidence supporting its efficacy. Future directions for this line of research were discussed as well. Daneault et al. [82] reviewed the neural pathways underlying the effects of light on NIF functions and discussed eye and cerebral mechanisms associated with aging which may affect NIF light sensitivity. They also reported on the results of investigations about pupillary constriction and cognitive brain sensitivity to light in the course of normal aging.

\section{Future prospects for the research on nonvisual effects of light in the field of physiological anthropology}

ipRGCs and melanopsin were discovered in the mammalian retina in the early 2000s. Studies on the nonvisual effects of light have become more popular since then. New findings in the field of photobiology, genetics, and other related disciplines are expected to prompt further developments in the research on the nonvisual effects of light in the field of physiological anthropology. Recently, it has been suggested that ipRGCs are involved not only in nonvisual functions, but also in visual functions [83-86]. Intriguingly, a small population of M1 ipRGCs (one of the five ipRGC subtypes) has intraretinal axon collaterals that project toward the outer retina and transmit luminance signals retrogradely, influencing retinal light adaptation [86]. Of particular interest has been observations that cone-derived color signals may influence nonvisual responses to light, such as circadian entrainment $[87,88]$ and PLR [89-91]. Woelders et al. [91] have demonstrated in 2018 that $\mathrm{M}$ - and S-cones provide inhibitory input to the pupillary control system, whereas L-cones and melanopsin response present an excitatory role. These findings support a subadditive response to light, where the effects of blue light are reduced by green or polychromatic light exposure [60, 63, 92, 93].

One of the main goals of the research in physiological anthropology is to build a truly adapted artificial environment based on the biological characteristics of human beings. In the early 1990s, Sato et al. [13-15] explored and provided supporting experimental evidence in regard to the disadvantages of using high color temperature lights. These studies were the main driving force of all subsequent research on the nonvisual effects of light. The results of these studies may have informed, for example, the current choices for using low color temperature lights in living rooms and bedrooms, and suggested the appropriate timing for light exposure in order to achieve sleep improvements and a healthy life. Furthermore, these results can also be used to define the wavelength of illumination and the transparency characteristics of the eyeglasses needed to achieve enough adaptability for daily activities. Once further knowledge is obtained, the potential applications of this research in daily life may be enormous.

\section{Conclusion}

Following a seminal study by Sato and colleagues on the effects of color temperature on human physiology in the early 1990s, research on the nonvisual effect of light in the field of physiological anthropology began and has increased over time. The discovery of ipRGC in the 
mammalian retina in the early 2000 s has renewed the scientific interest in this topic, and many interesting findings have been obtained also in the field of physiological anthropology since then.

The influences of color temperature of light exposed before sleep were studied. Detrimental effects of high color temperature light before sleep were found. Many other significant effects of color temperature of light were also found in other aspects of physiological anthropology, such as body temperature regulation, taste threshold, brain functions, or electrogastrogram study. Many studies on the light intensity have been conducted also in the field of physiological anthropology. Works on light intensity have mostly examined effects on the circadian rhythm and on the CNS and ANS activities, PLR, nocturnal melatonin suppression, and body temperature regulation. Studies on the influence of monochromatic light have been numerous, especially in recent years. Their main focus was the circadian rhythm and the pupillary constriction. A few other studies have examined the effects of monochromatic light on HRV, ERG, time sense, event-related potentials, and body temperature regulation. Quite a few papers were categorized into others. Some of them are comparative studies of the efficiency of carbohydrate absorption between Japan and Poland. One study also explored the relation between seasonal variations in melatonin secretion and surrounding light conditions. A genetic association study evaluated effects of genotype groups for one polymorphism in the melanopsin gene on sleep/wake timing.

Recently, it has been suggested that ipRGC may be involved not only in nonvisual functions, but also in visual functions. Moreover, it has been shown that cone-derived color signals influence nonvisual responses. Prompted by these new findings, research on the nonvisual effects of light in the field of physiological anthropology is expected to develop. This knowledge will be paramount to better understand how current artificial environments may affect human beings. From there, informed strategies of intervention may be derived to reduce potential detrimental effects, while preserving the benefits of many of these modern daily life achievements.

\section{Abbreviations}

AAC: Alpha attenuation coefficient; ANS: Autonomic nervous system; CIZ: Core interthreshold zone; CNS: Central nervous system; CNV: Contingent negative variation; EEG: Electroencephalogram; EGG: Electrogastrogram; ERG: Electroretinogram; HRV: Heart rate variability; ipRGC: Intrinsically photosensitive retinal ganglion cell; ISI: Inter-stimulus interval; JPA: Journal of Physiological Anthropology; M-E score: Morningness-Eveningness score; mRGC: Melanopsin-expressing retinal ganglion cell; NIF: Non-image-forming; PLR: Pupillary light reflex/response

\section{Availability of data and materials}

All data generated or analyzed during this study are included in this published article.
Authors' contributions

TK and SL contributed to the research, writing, and editing of this manuscript. Both authors read and approved the final manuscript.

Ethics approval and consent to participate

Not applicable

Consent for publication

Not applicable

Competing interests

The authors declare that they have no competing interests.

\section{Publisher's Note}

Springer Nature remains neutral with regard to jurisdictional claims in published maps and institutional affiliations.

\section{Author details}

${ }^{1}$ Graduate School of Engineering, Chiba University, 1-33 Yayoi-cho, Inage-ku, Chiba 263-8522, Japan. ${ }^{2}$ Center for Environment, Health and Sciences, Chiba University, 6-2-1 Kashiwanoha, Kashiwa, Chiba 277-0882, Japan.

Received: 1 October 2018 Accepted: 4 December 2018

Published online: 22 January 2019

References

1. Rockwell NC, Lagarias JC. A brief history of phytochromes. ChemPhysChem. 2010;11:1172-80

2. Possart A, Fleck C, Hiltbrunner A. Shedding (far-red) light on phytochrome mechanisms and responses in land plants. Plant Sci. 2014;217-218:36-46.

3. Lagman D, Ocampo Daza D, Widmark J, Abalo XM, Sundstrom G, Larhammar D. The vertebrate ancestral repertoire of visual opsins, transducin alpha subunits and oxytocin/vasopressin receptors was established by duplication of their shared genomic region in the two rounds of early vertebrate genome duplications. BMC Evol Biol. 2013;13:238.

4. Borges R, Johnson WE, O'Brien SJ, Gomes C, Heesy CP, Antunes A. Adaptive genomic evolution of opsins reveals that early mammals flourished in nocturnal environments. BMC Genomics. 2018;19:121.

5. Jacobs GH. Primate photopigments and primate color vision. Proc Natl Acad Sci U S A. 1996;93:577-81.

6. Imamoto Y, Shichida Y. Cone visual pigments. Biochim Biophys Acta. 2014; 1837:664-73.

7. Berson DM, Dunn FA, Takao M. Phototransduction by retinal ganglion cells that set the circadian clock. Science. 2002:295:1070-3.

8. Peirson SN, Halford S, Foster RG. The evolution of irradiance detection: melanopsin and the non-visual opsins. Philos Trans R Soc Lond Ser B Biol Sci. 2009;364:2849-65.

9. Bellingham J, Foster RG. Opsins and mammalian photoentrainment. Cell Tissue Res. 2002;309:57-71.

10. lyengar B. The melanocyte photosensory system in the human skin. Springerplus. 2013;2:158.

11. Provencio I, Jiang G, De Grip WJ, Hayes WP, Rollag MD. Melanopsin: an opsin in melanophores, brain, and eye. Proc Natl Acad Sci U S A. 1998;95:340-5.

12. Sato M. The development of conceptual framework in physiological anthropology. J Physiol Anthropol Appl Hum Sci. 2005;24:289-95.

13. Deguchi T, Sato M. The effect of color temperature of lighting sources on mental activity level. Ann Physiol Anthropol. 1992;11:37-43.

14. Kobayashi H, Sato M. Physiological responses to illuminance and color temperature of lighting. Ann Physiol Anthropol. 1992:11:45-9.

15. Mukae $H$, Sato $M$. The effect of color temperature of lighting sources on the autonomic nervous functions. Ann Physiol Anthropol. 1992;11:533-8.

16. Kruithof AA. Tubular luminescence lamps for general illumination. Phillips Tech Rev. 1941;6:65-73.

17. Foster RG, Provencio I, Hudson D, Fiske S, De Grip W, Menaker M. Circadian photoreception in the retinally degenerate mouse (rd/rd). J Comp Physiol A. 1991:169:39-50.

18. Hattar S, Liao HW, Takao M, Berson DM, Yau KW. Melanopsin-containing retinal ganglion cells: architecture, projections, and intrinsic photosensitivity. Science. 2002;295:1065-70.

19. Panda S, Provencio I, Tu DC, Pires SS, Rollag MD, Castrucci AM, Pletcher MT, Sato TK, Wiltshire T, Andahazy M, et al. Melanopsin is required for 
non-image-forming photic responses in blind mice. Science. 2003;301: $525-7$.

20. Qiu X, Kumbalasiri T, Carlson SM, Wong KY, Krishna V, Provencio I, Berson DM. Induction of photosensitivity by heterologous expression of melanopsin. Nature. 2005;433:745-9.

21. Lucas RJ, Hattar S, Takao M, Berson DM, Foster RG, Yau KW. Diminished pupillary light reflex at high irradiances in melanopsin-knockout mice. Science. 2003;299:245-7.

22. Provencio I, Rodriguez IR, Jiang G, Hayes WP, Moreira EF, Rollag MD. A novel human opsin in the inner retina. J Neurosci. 2000;20:600-5.

23. La Morgia C, Ross-Cisneros FN, Hannibal J, Montagna P, Sadun AA, Carelli V. Melanopsin-expressing retinal ganglion cells: implications for human diseases. Vis Res. 2011;51:296-302.

24. Morita T, Tokura H. Effects of lights of different color temperature on the nocturnal changes in core temperature and melatonin in humans. Appl Hum Sci. 1996;15:243-6.

25. Kozaki T, Kitamura S, Higashihara Y, Ishibashi K, Noguchi H, Yasukouchi A. Effect of color temperature of light sources on slow-wave sleep. J Physiol Anthropol Appl Hum Sci. 2005;24:183-6.

26. Ishibashi K, Kitamura S, Kozaki T, Yasukouchi A. Inhibition of heart rate variability during sleep in humans by $6700 \mathrm{~K}$ pre-sleep light exposure. J Physiol Anthropol. 2007;26:39-43.

27. Noguchi H, Sakaguchi T. Effect of illuminance and color temperature on lowering of physiological activity. Appl Hum Sci. 1999;18:117-23.

28. Yasukouchi A, Yasukouchi Y, Ishibashi K. Effects of color temperature of fluorescent lamps on body temperature regulation in a moderately cold environment. J Physiol Anthropol Appl Hum Sci. 2000;19:125-34.

29. Katsuura $T$, Jin $X$, Baba $Y$, Shimomura $Y$, Iwanaga K. Effects of color temperature of illumination on physiological functions. J Physiol Anthropol Appl Hum Sci. 2005;24:321-5.

30. Jin $X$, Katsuura $T$, Iwanaga $K$, Shimomura $Y$, Inoie $M$. The influence of taste stimuli and illumination on electrogastrogram measurements. J Physiol Anthropol. 2007;26:191-5.

31. Yasukouchi A, Ishibashi K. Non-visual effects of the color temperature of fluorescent lamps on physiological aspects in humans. J Physiol Anthropol Appl Hum Sci. 2005;24:41-3.

32. Lewy AJ, Wehr TA, Goodwin FK, Newsome DA, Markey SP. Light suppresses melatonin secretion in humans. Science. 1980;210:1267-9.

33. Park SJ, Tokura H. Effects of different light intensities during the daytime on circadian rhythm of core temperature in humans. Appl Hum Sci. 1998;17:253-7.

34. Kanikowska D, Hirata Y, Hyun K, Tokura H. Acute phase proteins, body temperature and urinary melatonin under the influence of bright and dim light intensities during the daytime. J Physiol Anthropol Appl Hum Sci. 2001:20:333-8.

35. Wakamura T, Tokura H. Influence of bright light during daytime on sleep parameters in hospitalized elderly patients. J Physiol Anthropol Appl Hum Sci. 2001;20:345-51.

36. Yokoi M, Aoki K, Shimomura Y, Iwanaga K, Katsuura T. Effect of bright light on EEG activities and subjective sleepiness to mental task during nocturnal sleep deprivation. J Physiol Anthropol Appl Hum Sci. 2003;22:257-63.

37. Higuchi S, Motohashi Y, Maeda T, Ishibashi K. Relationship between individual difference in melatonin suppression by light and habitual bedtime. J Physiol Anthropol Appl Hum Sci. 2005;24:419-23.

38. Yokoi M, Aoki K, Shimomura Y, Iwanaga K, Katsuura T. Exposure to bright light modifies HRV responses to mental tasks during nocturnal sleep deprivation. J Physiol Anthropol. 2006;25:153-61.

39. Hyun KJ, Nishimura S, Tokura H. Influences of diurnal bright or dim light exposure on urine volume in humans. J Physiol Anthropol. 2006;25:189-92.

40. Hirota N, Sone $\mathrm{Y}$, Tokura H. Effect of evening exposure to bright or dim light after daytime bright light on absorption of dietary carbohydrates the following morning. J Physiol Anthropol. 2010;29:79-83.

41. Kozaki T, Toda N, Noguchi H, Yasukouchi A. Effects of different light intensities in the morning on dim light melatonin onset. J Physiol Anthropol. 2011;30:97-102

42. Fukushige H, Fukuda Y, Tanaka M, Inami K, Wada K, Tsumura Y, Kondo M, Harada T, Wakamura T, Morita T. Effects of tryptophan-rich breakfast and light exposure during the daytime on melatonin secretion at night. J Physiol Anthropol. 2014;33:33.

43. Kozaki T, Kubokawa A, Taketomi R, Hatae K. Effects of day-time exposure to different light intensities on light-induced melatonin suppression at night. J Physiol Anthropol. 2015;34:27.
44. Nagashima S, Yamashita M, Tojo C, Kondo M, Morita T, Wakamura T. Can tryptophan supplement intake at breakfast enhance melatonin secretion at night? J Physiol Anthropol. 2017;36:20.

45. Higuchi S, Watanuki S, Yasukouchi A, Sato M. Effects of changes in arousal level by continuous light stimulus on contingent negative variation (CNV). Appl Hum Sci. 1997;16:55-60.

46. Noguchi H, Sakaguchi T, Sato M. Physiological effects of sudden change in illuminance during dark-adapted state. Appl Hum Sci. 1999;18:109-14.

47. Yoshinaga N, Fujita M, Tanaka YL, Nemoto S. Effects of changing illuminance on somatosensory function. J Physiol Anthropol. 2011;30:141-6.

48. Yasukouchi A, Hazama T, Kozaki T. Variations in the light-induced suppression of nocturnal melatonin with special reference to variations in the pupillary light reflex in humans. J Physiol Anthropol. 2007;26:113-21.

49. Kakitsuba N, Mekjavic IB, Katsuura T. The effect of season and light intensity on the core interthreshold zone. J Physiol Anthropol. 2011:30:161-7.

50. Schobersberger W, Blank C, Hanser F, Griesmacher A, Canazei M, Leichtfried $V$. Impact of a single, short morning bright light exposure on tryptophan pathways and visuo- and sensorimotor performance: a crossover study. J Physiol Anthropol. 2018;37:12.

51. Kim HE, Tokura H. Influence of light intensities on dressing behavior in elderly people. J Physiol Anthropol Appl Hum Sci. 2000;19:13-9.

52. Tokura H, Kim HE. How does light intensity influence evening dressing behavior in the cold? J Physiol Anthropol Appl Hum Sci. 2005;24:37-40.

53. Morita T, Tokura H, Wakamura T, Park SJ, Teramoto Y. Effects of the morning irradiation of light with different wavelengths on the behavior of core temperature and melatonin in humans. Appl Hum Sci. 1997;16:103-5.

54. Morita $\mathrm{T}$, Tokura $\mathrm{H}$. The influence of different wavelengths of light on human biological rhythms. Appl Hum Sci. 1998;17:91-6.

55. An M, Huang J, Shimomura Y, Katsuura T. Time-of-day-dependent effects of monochromatic light exposure on human cognitive function. J Physiol Anthropol. 2009;28:217-23.

56. Higuchi S, Fukuda T, Kozaki T, Takahashi M, Miura N. Effectiveness of a redvisor cap for preventing light-induced melatonin suppression during simulated night work. J Physiol Anthropol. 2011;30:251-8.

57. Kozaki T, Hidaka Y, Takakura JY, Kusano Y. Suppression of salivary melatonin secretion under $100-\mathrm{Hz}$ flickering and non-flickering blue light. J Physiol Anthropol. 2018;37:23.

58. Katsuura T, Ochiai Y, Senoo T, Lee S, Takahashi Y, Shimomura Y. Effects of blue pulsed light on human physiological functions and subjective evaluation. J Physiol Anthropol. 2012;31:23.

59. Lee SI, Hida A, Tsujimura S, Morita T, Mishima K, Higuchi S. Association between melanopsin gene polymorphism (1394T) and pupillary light reflex is dependent on light wavelength. J Physiol Anthropol. 2013;32:16.

60. Lee S, Ishibashi S, Shimomura Y, Katsuura T. Effect of simultaneous exposure to extremely short pulses of blue and green light on human pupillary constriction. J Physiol Anthropol. 2016;35:20.

61. Dai Q, Uchiyama Y, Lee S, Shimomura Y, Katsuura T. Effect of quantity and intensity of pulsed light on human non-visual physiological responses. J Physiol Anthropol. 2017;36:22.

62. Lee S, Muto N, Shimomura Y, Katsuura T. Human pupillary light reflex during successive irradiation with 1-ms blue- and green-pulsed light. J Physiol Anthropol. 2017;36:37.

63. Lee S, Uchiyama Y, Shimomura Y, Katsuura T. Subadditive responses to extremely short blue and green pulsed light on visual evoked potentials, pupillary constriction and electroretinograms. J Physiol Anthropol. 2017:36:39.

64. Yuda E, Ogasawara H, Yoshida Y, Hayano J. Suppression of vagal cardiac modulation by blue light in healthy subjects. J Physiol Anthropol. 2016;35:24.

65. Yuda E, Ogasawara H, Yoshida Y, Hayano J. Enhancement of autonomic and psychomotor arousal by exposures to blue wavelength light: importance of both absolute and relative contents of melanopic component. J Physiol Anthropol. 2017;36:13

66. Yuda E, Ogasawara H, Yoshida Y, Hayano J. Exposure to blue light during lunch break: effects on autonomic arousal and behavioral alertness. J Physiol Anthropol. 2017;36:30.

67. Katsuura T, Yasuda T, Shimomura Y, Iwanaga K. Effects of monochromatic light on time sense for short intervals. J Physiol Anthropol. 2007;26:95-100

68. Fukuda Y, Higuchi S, Yasukouchi A, Morita T. Distinct responses of cones and melanopsin-expressing retinal ganglion cells in the human electroretinogram. J Physiol Anthropol. 2012;31:20.

69. Kakitsuba N, Mekjavic IB, Katsuura T. The core interthreshold zone during exposure to red and blue light. J Physiol Anthropol. 2013;32:6. 
70. Yoto A, Katsuura T, Iwanaga K, Shimomura Y. Effects of object color stimuli on human brain activities in perception and attention referred to EEG alpha band response. J Physiol Anthropol. 2007;26:373-9.

71. Tsumura $Y$, Hirota N, Tokura H, Sone $Y$, Lesinski F, Rutkowska D, Barinow-Wojewodzki A. Comparison of carbohydrate digestion between Japanese and Polish healthy subjects. J Physiol Anthropol Appl Hum Sci. 2005;24:507-9.

72. Ueno-Towatari T, Norimatsu K, Blazejczyk K, Tokura H, Morita T. Seasonal variations of melatonin secretion in young females under natural and artificial light conditions in Fukuoka, Japan. J Physiol Anthropol. 2007;26:209-15.

73. Nakade M, Takeuchi H, Taniwaki N, Noji T, Harada T. An integrated effect of protein intake at breakfast and morning exposure to sunlight on the circadian typology in Japanese infants aged 2-6 years. J Physiol Anthropol. 2009;28:239-45.

74. Nakade M, Akimitsu O, Wada K, Krejci M, Noji T, Taniwaki N, Takeuchi H, Harada T. Can breakfast tryptophan and vitamin B6 intake and morning exposure to sunlight promote morning-typology in young children aged 2 to 6 years? J Physiol Anthropol. 2012:31:11.

75. Tanaka M, Hosoe K, Hamada T, Morita T. Change in sleep state of the elderly before and after cataract surgery. J Physiol Anthropol. 2010;29:219-24.

76. Lee SI, Hida A, Kitamura S, Mishima K, Higuchi S. Association between the melanopsin gene polymorphism OPN4*lle394Thr and sleep/wake timing in Japanese university students. J Physiol Anthropol. 2014;33:9.

77. Adamsson M, Laike T, Morita T. Annual variation in daily light exposure and circadian change of melatonin and cortisol concentrations at a northern latitude with large seasonal differences in photoperiod length. J Physiol Anthropol. 2017;36:6.

78. Kuller R. The influence of light on circarhythms in humans. J Physiol Anthropol Appl Hum Sci. 2002;21:87-91.

79. Yasukouchi A. A physio-anthropological approach in evaluation of human adaptability to living environment: in the case of artificial light environment. J Physiol Anthropol Appl Hum Sci. 2005;24:307-12.

80. Hanifin JP, Brainard GC. Photoreception for circadian, neuroendocrine, and neurobehavioral regulation. J Physiol Anthropol. 2007;26:87-94.

81. Crowley SK, Youngstedt SD. Efficacy of light therapy for perinatal depression: a review. J Physiol Anthropol. 2012;31:15.

82. Daneault V, Dumont M, Masse E, Vandewalle G, Carrier J. Light-sensitive brain pathways and aging. J Physiol Anthropol. 2016;35:9.

83. Zhang DQ, Wong KY, Sollars PJ, Berson DM, Pickard GE, McMahon DG. Intraretinal signaling by ganglion cell photoreceptors to dopaminergic amacrine neurons. Proc Natl Acad Sci U S A. 2008;105:14181-6.

84. Zhang DQ, Belenky MA, Sollars PJ, Pickard GE, McMahon DG. Melanopsin mediates retrograde visual signaling in the retina. PLoS One. 2012;7:e42647.

85. Atkinson CL, Feng J, Zhang DQ. Functional integrity and modification of retinal dopaminergic neurons in the rd1 mutant mouse: roles of melanopsin and GABA. J Neurophysiol. 2013;109:1589-99.

86. Prigge CL, Yeh PT, Liou NF, Lee CC, You SF, Liu LL, McNeill DS, Chew KS, Hattar S, Chen SK, et al. M1 ipRGCs influence visual function through retrograde signaling in the retina. J Neurosci. 2016;36:7184-97.

87. Walmsley L, Hanna L, Mouland J, Martial F, West A, Smedley AR, Bechtold DA, Webb AR, Lucas RJ, Brown TM. Colour as a signal for entraining the mammalian circadian clock. PLoS Biol. 2015;13:e1002127.

88. Spitschan M, Lucas RJ, Brown TM. Chromatic clocks: color opponency in non-image-forming visual function. Neurosci Biobehav Rev. 2017;78:24-33.

89. Spitschan M, Jain S, Brainard DH, Aguirre GK. Opponent melanopsin and S-cone signals in the human pupillary light response. Proc Natl Acad Sci U S A. 2014;111:15568-72

90. Hayter EA, Brown TM. Additive contributions of melanopsin and both cone types provide broadband sensitivity to mouse pupil control. BMC Biol. 2018;16:83.

91. Woelders T, Leenheers T, Gordijn MCM, Hut RA, Beersma DGM, Wams EJ. Melanopsin- and L-cone-induced pupil constriction is inhibited by S- and M-cones in humans. Proc Natl Acad Sci U S A. 2018:115:792-7.

92. Figueiro MG, Bierman A, Rea MS. Retinal mechanisms determine the subadditive response to polychromatic light by the human circadian system. Neurosci Lett. 2008;438:242-5

93. Revell VL, Barrett DC, Schlangen LJ, Skene DJ. Predicting human nocturnal nonvisual responses to monochromatic and polychromatic light with a melanopsin photosensitivity function. Chronobiol Int. 2010:27:1762-77.

\section{Ready to submit your research? Choose BMC and benefit from:}

- fast, convenient online submission

- thorough peer review by experienced researchers in your field

- rapid publication on acceptance

- support for research data, including large and complex data types

- gold Open Access which fosters wider collaboration and increased citations

- maximum visibility for your research: over $100 \mathrm{M}$ website views per year

At BMC, research is always in progress.

Learn more biomedcentral.com/submissions 